Article

\title{
Delineating the Molecular Basis of the Calmodulin-bMunc13-2 Interaction by Cross-Linking/Mass Spectrometry-Evidence for a Novel CaM Binding Motif in bMunc13-2
}

\author{
Christine Piotrowski ${ }^{1}$, Rocco Moretti ${ }^{2}$, Christian H. Ihling ${ }^{1}$, André Haedicke ${ }^{3,+}$, \\ Thomas Liepold ${ }^{4}$, Noa Lipstein ${ }^{5}$ (D) Jens Meiler ${ }^{2} \mathbb{D}$, Olaf Jahn ${ }^{4, *}$ (D) and Andrea Sinz ${ }^{1, *}$ \\ 1 Department of Pharmaceutical Chemistry and Bioanalytics, Institute of Pharmacy, \\ Charles Tanford Protein Center, Martin Luther University Halle-Wittenberg, D-06120 Halle/Saale, Germany; \\ christine.piotrowski@pharmazie.uni-halle.de (C.P.); christian.ihling@pharmazie.uni-halle.de (C.H.I.) \\ 2 Center for Structural Biology, Department of Chemistry, Vanderbilt University, Nashville, TN 37221, USA; \\ rmorettiase@gmail.com (R.M.); jens.meiler@vanderbilt.edu (J.M.) \\ 3 Biophysical Chemistry, Institute of Chemistry, Martin Luther University Halle-Wittenberg, \\ D-06120 Halle/Saale, Germany; andre.haedicke@chemie.uni-halle.de \\ 4 Proteomics Group, Max Planck Institute of Experimental Medicine, D-37075 Göttingen, Germany; \\ liepold@em.mpg.de \\ 5 Department of Molecular Neurobiology, Max Planck Institute of Experimental Medicine, D-37075 Göttingen, \\ Germany; lipstein@em.mpg.de \\ * Correspondence: jahn@em.mpg.de (O.J.); andrea.sinz@pharmazie.uni-halle.de (A.S.); \\ Tel.: +49-551-3899-313 (O.J.); +49-345-5525170 (A.S.) \\ + Present address: Serumwerk Bernburg AG, D-06406 Bernburg, Germany.
}

Received: 21 November 2019; Accepted: 2 January 2020; Published: 7 January 2020

check for updates

\begin{abstract}
Exploring the interactions between the $\mathrm{Ca}^{2+}$ binding protein calmodulin $(\mathrm{CaM})$ and its target proteins remains a challenging task. Members of the Munc13 protein family play an essential role in short-term synaptic plasticity, modulated via the interaction with CaM at the presynaptic compartment. In this study, we focus on the bMunc13-2 isoform expressed in the brain, as strong changes in synaptic transmission were observed upon its mutagenesis or deletion. The CaM-bMunc13-2 interaction was previously characterized at the molecular level using short bMunc13-2-derived peptides only, revealing a classical 1-5-10 CaM binding motif. Using larger protein constructs, we have now identified for the first time a novel and unique CaM binding site in bMunc13-2 that contains an $\mathrm{N}$-terminal extension of a classical 1-5-10 CaM binding motif. We characterize this motif using a range of biochemical and biophysical methods and highlight its importance for the CaM-bMunc13-2 interaction.
\end{abstract}

Keywords: Munc13; calmodulin; cross-linking; mass spectrometry; protein-protein interaction

\section{Introduction}

$\mathrm{Ca}^{2+}$ acts as a key regulator of multiple cellular processes, such as cell proliferation, differentiation, apoptosis, and neurotransmitter release $[1,2]$. Changes in $\mathrm{Ca}^{2+}$ concentrations are sensed by various proteins that either bind to $\mathrm{Ca}^{2+}$ itself or interact with $\mathrm{Ca}^{2+}$ binding proteins. A prominent $\mathrm{Ca}^{2+}$ binding protein is calmodulin (CaM), a small $17-\mathrm{kDa}$ acidic protein that is highly conserved in all eukaryotes and has an identical amino acid sequence in vertebrates [3,4]. CaM is a 148-amino acid protein arranged in two globular domains, each containing two $\mathrm{Ca}^{2+}$ binding EF-hands that are connected by a flexible linker [5-7]. CaM evolved to enable promiscuous target binding, requiring few specific interactions to interact with its more than 300 known target proteins [8-10]. Target binding occurs through a structural 
rearrangement of CaM, allowing hydrophobic amino acids of CaM to arrange into a binding pocket [11]. In $\mathrm{Ca}^{2+}$-dependent binding, targets adopt a basic amphiphilic helical structure where the hydrophobic anchor amino acids at specific positions define different $\mathrm{Ca}^{2+}$ binding motifs, such as 1-8-14 or 1-5-10 motifs [12]. $\mathrm{Ca}^{2+}$-independent IQ-motifs also feature a helical structure with a positive net charge, but with no or only low amphiphilicity [13]. Known CaM binding motifs are summarized in CaM target databases that aid in determining CaM interaction sites and in identifying novel CaM targets $[14,15]$.

At the presynaptic compartment, where synaptic vesicles (SVs) fuse with the plasma membrane to release neurotransmitter into the synaptic cleft, regulation of protein function by CaM binding plays a major role, with over 75 proteins proposed as CaM targets [16]. One such well-studied regulatory interaction is the $\mathrm{Ca}^{2+}$-dependent $\mathrm{CaM} / \mathrm{Munc13}$ interaction, which occurs after activity-induced $\mathrm{Ca}^{2+}$ influx. Munc13s are absolutely essential for the assembly of SNARE complexes [17,18], which link the SV membrane with the active zone plasma membrane [19]. This generates a pool of readily releasable SVs, where the molecular machinery is assembled in a form that allows a local and accurate neurotransmitter release in response to an action potential-evoked $\mathrm{Ca}^{2+}$-influx. The Munc13 protein family is composed of multiple members, four of which are expressed in the central nervous system (CNS) [20,21]. Munc13-1 is the dominant isoform expressed in the vast majority of CNS synapses [22], with some synapse types expressing additional isoforms. Hippocampal synapses co-express bMunc13-2 as the major Munc13-2 isoform [23]. It is derived as a splice isoform from the Unc13B gene, together with ubMunc13-2. In contrast, cerebellar synapses co-express primarily Munc13-3 with Munc13-1 [24]. All Munc13 isoforms share a conserved $C$-terminal domain structure composed of a C1 domain, a central C2 domain, a MUN domain, and a C-terminal C2 domain (Figure 1). Interestingly, the $N$-terminal domains are rather divergent and contain large unstructured regions, for which little functional information is available to date.

In line with the essential role of Munc13s in generating fusible SVs, Munc13-1 has recently been implicated in the determination of synaptic strength [25]. The role of Munc13-2 proteins, however, is still not fully understood. Evidence points to an involvement in the fine-tuning of synaptic efficacy and plasticity [26-29]. A major pathway that regulates synaptic plasticity via Munc13 proteins is the $\mathrm{Ca}^{2+} / \mathrm{CaM}$-dependent pathway. We have previously shown that all Munc13 isoforms contain a single, functional CaM binding site at a similar $N$-terminal position of their C1 domains [28] and that $\mathrm{Ca}^{2+} / \mathrm{CaM}$ binding to that site increases synaptic efficacy during activity. In bMunc13-2 and Munc13-3, another candidate CaM binding site was predicted and indeed shown to bind CaM at the level of peptides [28]. However, cosedimentation experiments with GST-bMunc13-2(367-780) and mutated variants thereof provided strong evidence that CaM binds in a 1:1 stoichiometry and that the further C-terminally located CaM binding site is the relevant one in the protein context [28]. The CaM/Munc13-1 interaction was studied in great detail at the functional level both in vitro [30] and in vivo [31], and at the structural level [32]. In particular, the canonical 1-5-8 CaM binding sites of the closely related isoforms Munc13-1 and ubMunc13-2 were shown to extend C-terminally, leading to the identification of a secondary hydrophobic contact point with CaM and thus to the definition of a novel 1-5-8-26 CaM binding motif [32]. Evidence from NMR experiments indicated that the elongated motif may mediate sequential CaM binding, as a potential mechanism for $\mathrm{Ca}^{2+}$-sensing over a broad concentration range. However, no such motif is present in bMunc13-2, raising the question of whether other elongated CaM binding sites exist in this isoform, particularly in view of the fact that bMunc13-2 exhibits the strongest CaM-dependent effects on synaptic plasticity in cultured hippocampal neurons [28].

We study the CaM/bMunc13-2 interaction in more detail by using larger bMunc13-2 constructs and elongated peptides, rather than minimal CaM-binding peptides. We applied an integrated approach based on chemical cross-linking/mass spectrometry (XL-MS), a method to investigate protein conformations and map protein-protein interactions [33-38] by covalently connecting functional groups of amino acid side chains that are in spatial proximity to a protein or a protein complex. Depending on the specific spacer length of the cross-linking reagents, mass spectrometric analysis reveals distance information for the covalently connected amino acids, which can be used to derive 
three-dimensional protein structures by computational modeling approaches [39-41]. We used XL-MS and complementary interaction analyses to show that the CaM binding to bMunc13-2 involves the $N$-terminal elongation of the previously defined CaM binding site. This elongated CaM-binding sequence is unique in bMunc13-2 and confers a substantially higher affinity for CaM binding. Our data underscore the importance of considering extended sequence motifs including core CaM binding domains and their flanking regions for a deeper understanding of CaM-dependent regulation of Munc13 proteins in the presynapse.

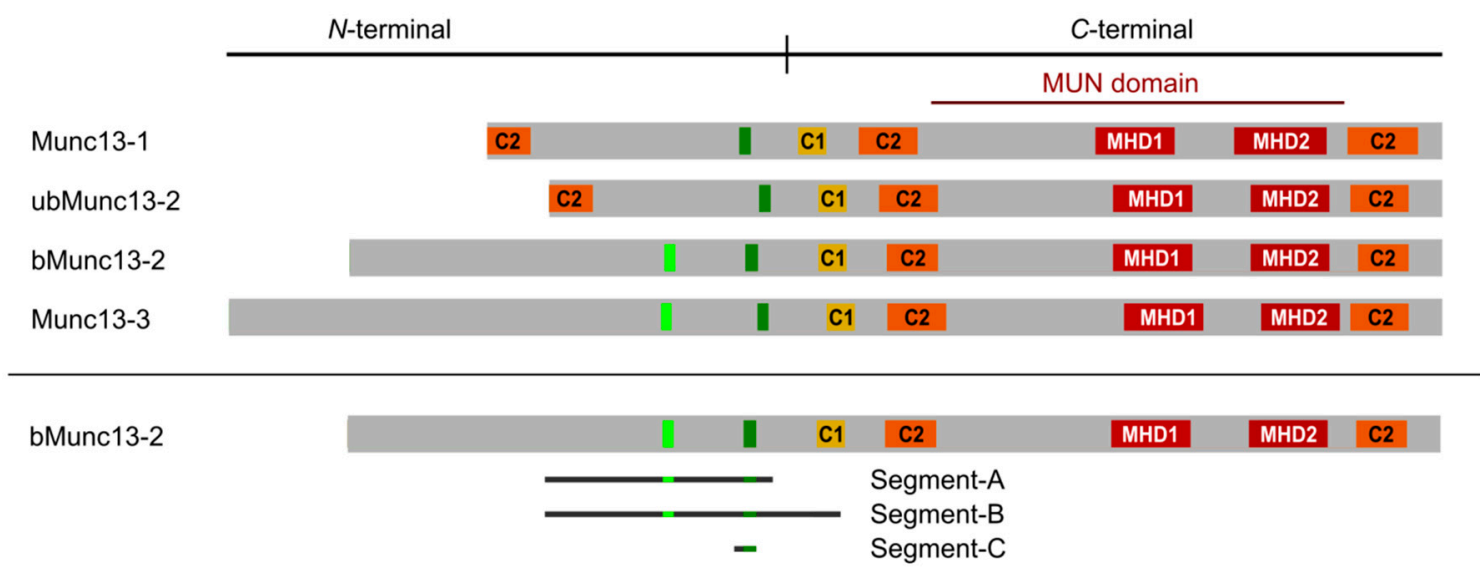

Figure 1. Domain structure of Munc13 proteins. All Munc13 isoforms share a conserved C-terminal priming unit and a central regulatory unit. Munc13-1 and ubMunc13-2 have a homologous $N$-terminal region containing an additional C2 domain. CaM-binding sites (dark green bars) are located in the regulatory unit. In bMunc13-2 and Munc13-3, another candidate CaM binding site (light green bars) was initially identified by computational methods, but found to be deficient of CaM-binding in the protein context [28]. C1 = diacylglycerol binding domain (yellow), $\mathrm{C} 2=\mathrm{Ca}^{2+}$-binding/protein interaction domain (orange), MHD = Munc homology domain (red). The bMunc13-2 segments investigated in this study are displayed. Segment-A (aa 367-780), segment-B (aa 367-903), and segment-C (aa 703/704-742).

\section{Materials and Methods}

\subsection{Materials}

CaM (bovine brain) was obtained from Athens Research (Athens, GA, USA). Cross-linkers were purchased from Thermo Fisher Scientific (Dreieich, Germany) (s-GMBS, BS ${ }^{3}$ and photo-Met) or synthesized in house (DSBU) [42]. The proteases trypsin (porcine) and GluC were obtained from Promega GmbH (Mannheim, Germany). Chemicals were obtained from Sigma Aldrich (Taufkirchen, Germany), Merck (Darmstadt, Germany), and VWR (Darmstadt, Germany). Prestained Protein Ladder was purchased from Thermo Fisher Scientific. For HPLC, solvents were LC-MS grade (VWR, Darmstadt, Germany). MilliQ water was produced by a TKA X-CAD system (Thermo Fisher Scientific, Niederelbert, Germany).

\subsection{Expression and Purification of Recombinant CaM and bMunc13-2 Segments}

Photo-Met labeled CaM (Figure S1) was expressed and purified as described previously [43]. Recombinant photo-Met-labeled CaM was stored at $-20^{\circ} \mathrm{C}$ in $20 \mathrm{mM}$ HEPES, $150 \mathrm{mM} \mathrm{NaCl}$ buffer (pH 7.2 at $25^{\circ} \mathrm{C}$ ). Recombinant rat bMunc13-2 segments (segment-A, aa 367-780; segment-B, aa 367-903; Figure S2) were expressed with $N$-terminal glutathione-S-transferase (GST) tag in the E. coli strain BL21(DE3) $c^{+}$RIL pGEX-4T-1. The E. coli strain was grown on TB medium to an $\mathrm{OD}_{600 \mathrm{~nm}}$ of 0.6 at $37^{\circ} \mathrm{C}$. Protein production was induced by adding IPTG to a final concentration of $0.1 \mathrm{mM}$. Cells were further grown at $18^{\circ} \mathrm{C}$ and harvested after incubation overnight. GST-tagged segments were enriched from cell extracts by GST affinity chromatography and strong cation exchange chromatography on an 
ÄKTA FPLC system (GE Healthcare Life Sciences, Freiburg, Germany). The GST tag was cleaved by thrombin $(0.08 \mathrm{mg} / \mathrm{mL}$ protein solution $)$ overnight at $6{ }^{\circ} \mathrm{C}$. Purified segments were stored at $-20{ }^{\circ} \mathrm{C}$ in $20 \mathrm{mM}$ HEPES, $300 \mathrm{mM} \mathrm{NaCl}, 0.5 \mathrm{mM}$ TCEP, $10 \%$ glycerol buffer (pH 7.2 at $25^{\circ} \mathrm{C}$ ). Segment-C and its variants were synthesized by standard solid phase peptide synthesis using fluorenylmethyloxycarbonyl (Fmoc) chemistry.

\section{3. (Photo-)Chemical Cross-Linking}

Cross-linking experiments were performed at $25^{\circ} \mathrm{C}$ with free $\mathrm{Ca}^{2+}$ concentrations ranging between $75 \mathrm{~nm}$ and $1 \mathrm{mM}$. Ca ${ }^{2+}$ levels were controlled using an EGTA chelating system [44].

Photo-cross-linking between photo-Met-labeled CaM and bMunc13-2 segments. Photo-cross-linking reactions were conducted in $20 \mathrm{mM}$ HEPES, $250 \mathrm{mM} \mathrm{NaCl}, 0.5 \mathrm{mM}$ TCEP, $7.5 \%$ glycerol buffer ( $\mathrm{pH} 7.2$ at $\left.25{ }^{\circ} \mathrm{C}\right)$. Photo-Met labeled CaM $(10 \mu \mathrm{M})$ was incubated in the respective $\mathrm{Ca}^{2+} / \mathrm{EGTA}$ buffer for $15 \mathrm{~min}$ at $25^{\circ} \mathrm{C}$. Afterwards, $10 \mu \mathrm{M}$ segment-A, $-\mathrm{B}$, or $-\mathrm{C}$ were added and the mixture was incubated for $60 \mathrm{~min}$ at $25^{\circ} \mathrm{C}$. To induce photo-cross-linking, the reaction mixtures were subjected to UV-A irradiation ( $360 \mathrm{~nm})$ at $8000 \mathrm{~mJ} / \mathrm{cm}^{2}$ in a home-built irradiation chamber [45].

Chemical cross-linking between CaM and bMunc13-2 segments. All reactions were conducted in $20 \mathrm{mM}$ HEPES, $230 \mathrm{mM} \mathrm{NaCl}, 0.5 \mathrm{mM}$ TCEP, $7.5 \%$ glycerol buffer ( $\mathrm{pH} 7.2$ at $25^{\circ} \mathrm{C}$ ) with CaM (bovine brain). For BS $3\left(\mathrm{D}_{0} / \mathrm{D}_{4}\right)$ and DSBU cross-linking, CaM $(10 \mu \mathrm{M})$ was incubated in the respective $\mathrm{Ca}^{2+} /$ EGTA buffer for $15 \mathrm{~min}$ at $25^{\circ} \mathrm{C}$. Afterwards, $10 \mu \mathrm{M}$ segment-A was added and incubated at $25^{\circ} \mathrm{C}$ for $60 \mathrm{~min}$, before the cross-linker was added to a final concentration of $1 \mathrm{mM}$. The cross-linking reaction was allowed to proceed for $60 \mathrm{~min}$ at $25^{\circ} \mathrm{C}$ before the reaction was quenched by adding $\mathrm{NH}_{4} \mathrm{HCO}_{3}$ or TRIS to a final concentration of $20 \mathrm{mM}$.

Cross-linking with s-GMBS was performed as a two-step reaction. In a first step, CaM was labeled with s-GMBS by mixing $60 \mu \mathrm{M} \mathrm{CaM}$ with a 50-fold molar excess of s-GMBS and incubation at $25^{\circ} \mathrm{C}$ for $60 \mathrm{~min}$. The reaction was quenched by the addition of $\mathrm{NH}_{4} \mathrm{HCO}_{3}(20 \mathrm{mM}$ final concentration) and excess cross-linker was removed by centrifugation with ZebaSpin desalting columns ( $7 \mathrm{kDa}$ cut-off, Thermo Scientific). After incubating $10 \mu \mathrm{M}$ s-GMBS-labeled CaM in a $\mathrm{Ca}^{2+} /$ EGTA buffer for $15 \mathrm{~min}$ at $25^{\circ} \mathrm{C}, 10 \mu \mathrm{M}$ segment-A was added and the cross-linking reaction of the sulfhydryl-reactive site of $\mathrm{s}-\mathrm{GMBS}$ was allowed to proceed for $60 \mathrm{~min}$ at $25^{\circ} \mathrm{C}$. The reaction was quenched by the addition of glutathione (20 mM final concentration).

\subsection{Enzymatic Digestion}

For in-solution digestion, the protein solution was mixed with a solution containing $8 \mathrm{M}$ urea and $400 \mathrm{mM} \mathrm{NH}_{4} \mathrm{HCO}_{3}$ at 1:3 $(v / v)$ ratio or $1.5 \%$ deoxycholate were added. The proteins were reduced with dithiothreitol (DTT) and alkylated with iodoacetamide. The solution was diluted with water 1:10 $(v / v)$, followed by the addition of GluC at a protein:enzyme ratio of $30: 1(w / w)$ and incubation at $30{ }^{\circ} \mathrm{C}$ overnight. Subsequently, trypsin was added at a protein:enzyme ratio of 30:1 (w/w) and digestion was performed at $37^{\circ} \mathrm{C}$ for $4 \mathrm{~h}$. In-gel digestion was performed by excising the bands of interest from the SDS-gel. Gel pieces were subjected to in-gel digestion as described previously using GluC/trypsin or AspN/trypsin as digestion enzymes [45].

\subsection{Liquid Chromatography/Mass Spectrometry (LC/MS)}

Peptide digestion mixtures were separated on an Ultimate 3000 RSLC NanoHPLC system (Thermo Fisher Scientific); precolumn: C8 reversed phase, Acclaim PepMap, $300 \mu \mathrm{m} \times 5 \mathrm{~mm}, 5 \mu \mathrm{m}, 100 \AA$ (Thermo Fisher Scientific) or C18 reversed phase, Acclaim PepMap, $300 \mu \mathrm{m} \times 5 \mathrm{~mm}, 5 \mu \mathrm{m}, 100 \AA$ (Thermo Fisher Scientific); separation column: C18 reversed-phase, Acclaim PepMap, $75 \mu \mathrm{m} \times 250 \mathrm{~mm}$, $2 \mu \mathrm{m}, 100 \AA$ (Thermo Fisher Scientific) or PicoFrit C18 nanospray column, $75 \mu \mathrm{m}$ ID, $10 \mu \mathrm{m}$ tip (New Objective), packed with ReproSil-Pur 120 C18-AQ, $1.9 \mu \mathrm{m}$. Peptide mixtures were washed on the precolumn with water containing $0.1 \%$ TFA for $15 \mathrm{~min}$, before the peptides were separated on the separation column using gradients from $1 \%$ to $35 \%$ (40\%) B (90 $\mathrm{min}$ ), $35 \%$ (40\%) to $85 \%$ B (5 min) 
followed by $85 \%$ B (5 min), with solvent A: $0.1 \%$ formic acid (FA) in water and solvent B: $0.08 \%$ FA in acetonitrile. The nano-HPLC system was directly coupled to a Fusion Tribrid Orbitrap mass spectrometer equipped with a Nanospray Flex Ion Source (Thermo Fisher Scientific, Bremen, Germany). Data were acquired using the data-dependent MS/MS mode, i.e., each high-resolution full-scan in the Orbitrap ( $m / z 300$ to $2000, R=120,000)$ was followed by high-resolution product ion scans in the orbitrap $(R=15,000)$ within $5 \mathrm{~s}$, starting with the most intense signal in the full-scan mass spectrum (isolation window $2 \mathrm{Th}$ ). Fragmentation was achieved by CID (35\% normalized collision energy), HCD ( $29 \%$ normalized collision energy) or stepped HCD (30\% $\pm 3 \%$ normalized collision energy). Dynamic exclusion (exclusion duration: $60 \mathrm{~s}$, exclusion window: $\pm 2 \mathrm{ppm}$ ) was enabled to allow detection of less abundant ions. Data acquisition was controlled with Xcalibur v3.0.63 (Thermo Fisher Scientific).

\subsection{Identification of Cross-Links}

Data were analyzed with the StavroX (v3.4.9 to v3.6.6) and MeroX (v1.4.12 to v1.6.6) software tools [46,47] (www.StavroX.com). Mass tolerances were 3 ppm (MS) and 10 ppm (MS/MS); the signal-to-noise ratio was set to 2.0. Cross-linking sites were specifically defined for each cross-linking principle (Figure S3): for photo-Met, all 20 proteinogenic amino acids were considered as potential reaction sites; for $\mathrm{BS}^{3}$ and $\mathrm{DSBU}$, lysines, serines, threonines, $\mathrm{N}$-termini [48] were considered as reaction sites; for s-GMBS, cysteines were included. For photo-Met, oxidation of Met, carbamidomethylation of Cys and exchange of Met with photo-Met were considered as variable modifications. For BS ${ }^{3}$ and DSBU, carbamidomethylation of Cys was set as static modification; for $\mathrm{BS}^{3}$, DSBU, and s-GMBS, oxidation of Met was set as variable modification. Three missed cleavage sites were allowed for each cleavage site. All cross-links were manually validated.

\subsection{Docking of CaM/bMunc13-2 Segment-C Complexes}

Details on the modeling process are described in Supplementary Material III (Supplementary Methods: Protocol capture page for modeling approach).

Models of the CaM/segment-C (aa 704-742) interaction were generated using the ROSETTA FlexPepDock ab initio application [49]. All steps were performed with ROSETTA 2015.31.58019 and ROSETTA 2016.32.58837. To enable the docking process, an $\alpha$-helical 3D-structure of the peptide was created by the BuildPeptide command and manually placed into the CaM structure derived from the PDB-file $2 \mathrm{O} 60$ (CaM/NO synthase peptide complex [50]) in PyMOL (v1.2r1). Input for the FlexPepDock application was the structure generated and a constraints file, which contained information about the expected localization of the CaM binding site of bMunc13-2 within CaM obtained from PDB structures 2KDU [32] and 2O60 [50]. The 10,000 models generated were rescored by the distance constraints obtained from the cross-linking experiments. Distance thresholds were set to $\leq 8 \AA$ for photo-Met and $\leq 40 \AA$ for DSBU [39]. A KofN selection (the best $K$ crosslinks from $N$ total; $K=5$ to $7 ; N=10$ ) was applied to allow for only a fraction of all cross-links fitting into one model. Afterwards, the models were clustered by atom_pair_constraints and sorted by the given re-weighted score and atom pair constraints. Models, which were found to satisfy 5, 6 as well as 7 of 10 cross-links, were selected and subjected to relaxation to prevent structural tensions. Subsequently, the five top-scoring models were applied to two additional cycles of rescoring and relaxation. The resulting models were manually inspected and two were identified as the final models.

\subsection{Native Electrospray Ionization Mass Spectrometry}

Native ESI-MS was performed on a high-mass Q-TOF2 mass spectrometer (Waters Micromass/MS Vision, Almere, Netherlands). The $10 \mu \mathrm{M}$ solutions of CaM and bMunc13-2 segment- $\mathrm{A}$, as well as a mixture of $3.33 \mu \mathrm{M} \mathrm{CaM}$ and $12 \mu \mathrm{M}$ segment-C, were prepared in $300 \mathrm{mM}$ ammonium acetate ( $\mathrm{pH} 7.0)$ using centrifugation units ( 3 or $10 \mathrm{kDa}$ cut-off, Amicon Ultra, $0.5 \mathrm{~mL}$, Millipore, Darmstadt, Germany). Data were acquired using a capillary voltage of $1.38 \mathrm{kV}$, a cone voltage of $160-180 \mathrm{~V}$, an extraction cone voltage of 5-10 V, and collision energies between 10 and $80 \mathrm{~V}$. 


\subsection{Isothermal Titration Calorimetry}

Isothermal titration calorimetry (ITC) measurements were performed at $25^{\circ} \mathrm{C}$ using a MicroCal VP-ITC system (MicroCal Inc., Northampton, MA) with $20 \mathrm{mM}$ HEPES, $230 \mathrm{mM} \mathrm{NaCl}, 0.5 \mathrm{mM}$ TCEP buffer $\left(\mathrm{pH} 7.2 ; 25^{\circ} \mathrm{C}\right)$. All solutions were degassed for $10 \mathrm{~min}$ under vacuum prior to measurement. A CaM solution $(4 \mu \mathrm{M})$ was present in the sample cell, while bMunc13-2 segment-C solution $(80 \mu \mathrm{M})$ was applied via syringe. For ITC measurements, segment- $C$ was injected in $5-\mu \mathrm{L}$ steps into the sample cell. As a reference, the heat of dilution was determined by injection of the peptide into the ITC buffer without CaM that was subtracted afterwards from the titration curve. Data analysis was performed by the ITC module implemented in Origin 6.0 (MicroCal Inc., Northampton, MA, USA).

\subsection{Photoaffinity Labeling-Based Competition Assay}

Photoreactive peptides were synthesized by Fmoc chemistry using the amino acid derivative Fmoc-para-benzoylphenylalanine (Bpa, Novabiochem). Photoaffinity labeling (PAL)-based competition experiments with recombinant $\mathrm{CaM}$ were performed and analyzed as described previously [28]. Briefly, CaM $(5 \mu \mathrm{M})$ and segment-C-Bpa $(5 \mu \mathrm{M})$ or segment-CS-Bpa $(5 \mu \mathrm{M})$ were incubated in $50 \mathrm{mM}$ HEPES (pH 7.2), $150 \mathrm{mM} \mathrm{KCl}, 5 \mathrm{mM}$ DTT for $2 \mathrm{~h}$ at RT in the dark with $\left(200 \mu \mathrm{M} \mathrm{Ca}^{2+}\right)$ or without $(2 \mathrm{mM}$ EGTA) calcium, and in the presence of increasing concentrations $(0-250 \mu \mathrm{M})$ of competitor peptides. Following UV irradiation, photoadduct formation was visualized and quantified by SDS-PAGE and linear matrix-assisted laser desorption/ionization-time-of-flight-mass spectrometry (MALDI-TOF-MS).

\subsection{Surface Plasmon Resonance Spectroscopy}

The CaM-bMunc13-2 segment-C interaction was studied by surface plasmon resonance (SPR) using an MP-SPR Navi 200 OTSO instrument (BioNavis, Tampere, Finland). All steps were performed with a flow rate of $30 \mu \mathrm{L} / \mathrm{min}$ and sensograms were recorded at a laser wavelength of $670 \mathrm{~nm}$. First, $2 \mu \mathrm{M}$ of tetrameric streptavidin, reconstituted in $20 \mathrm{mM}$ HEPES pH 7.5, was injected twice to be immobilized on a PEG-biotin sensor chip (Cenibra $\mathrm{GmbH}$, Bramsche, Germany). Photo-Met-labeled CaM was biotinylated in 20 mM HEPES, pH 6.5, with a 10-fold molar excess EZ-Link NHS-LC-biotin (Thermo Fisher Scientific, Dreieich, Germany) over protein concentration. The reaction was performed at $25^{\circ} \mathrm{C}$ for $1 \mathrm{~h}$ and quenched by the addition of $\mathrm{NH}_{4} \mathrm{HCO}_{3}(20 \mathrm{mM}$ final concentration). Afterwards, excess of biotinylation reagent was removed by ZebaSpin desalting columns ( $7 \mathrm{kDa}$ cut-off) performing a buffer exchange to $20 \mathrm{mM}$ HEPES, $\mathrm{pH} 7.5$, at the same time. The biotinylation was verified by MALDI-TOF-MS (Figure S4). $7 \mu \mathrm{M}$ of biotinylated CaM was injected to immobilize the CaM onto streptavidin in the sample cell; CaM was collected and reinjected twice to maximize the amount of immobilized protein. Segment-C and its variants were dissolved in $20 \mathrm{mM}$ HEPES, $230 \mathrm{mM} \mathrm{NaCl}$, $0.5 \mathrm{mM}$ TCEP buffer ( $\mathrm{pH} 7.2 ; 25^{\circ} \mathrm{C}$ ). The interaction was studied by injection of $0.1-1 \mu \mathrm{M}$ segment-C variant. $\mathrm{K}_{\mathrm{D}}$ values were obtained using the TraceDrawer software (Ridgeview, Vänge, Sweden).

\section{Results}

\section{1. $\mathrm{Ca}^{2+}$-Dependent Formation of a Stoichiometric Complex between CaM and bMunc13-2}

As bMunc13-2 is a $>220 \mathrm{kDa}$ multi-domain protein that is difficult to express, two different constructs and a synthetic peptide, all derived from its $\mathrm{N}$-terminal region, were employed as proxies for the intact protein to study the CaM-bMunc13-2 interaction. Segment-A (aa 367-780) contains both the established CaM binding site and the predicted candidate CaM binding site, of which only the former was shown to bind to CaM at the protein level. Segment-B (aa 367-903) contains the sequence of segment- $A$ and is $C$-terminally elongated to include the $C 1$ domain. Segment- $C$ (aa 704-742) spans only the established CaM binding site and the $\sim 15$ aa stretch located directly $N$-terminal of it (Figure 1). As a starting point for investigating complex formation, we expressed segment-A with an $N$-terminal GST-tag, a construct successfully used in previous cosedimentation experiments [28]. 
For our cross-linking studies, the GST-tag was removed via its thrombin cleavage site to yield the native bMunc13-2 segment-A with 12 additional $N$-terminal amino acids originating from the multiple cloning site of the plasmid (Figure S2A).

With the aim of identifying the effective $\mathrm{Ca}^{2+}$ concentration range in vitro, we first studied the $\mathrm{Ca}^{2+}$ dependence of complex formation between $\mathrm{CaM}$ and segment-A by photo-cross-linking experiments with photo-Met-labeled CaM [43]. CaM contains nine methionines (Figure S1) that were all found to be replaced by photo-Met with incorporation rates of up to 30\% [43]. The artificial amino acid photo-Met contains a diazirine moiety that is activated upon UV-A irradiation $(\sim 360 \mathrm{~nm})$, resulting in the formation of a covalent bond between amino acids in close spatial proximity (Figure S3A) [51]. When we evaluated complex formation between photo-Met-labeled CaM and segment-A at different $\mathrm{Ca}^{2+}$ concentrations (Figure 2A), we identified $750 \mathrm{nM} \mathrm{Ca}^{2+}$ as a low, but already saturating concentration. For all later experiments aiming at structural characterization of the complex, we chose this low concentration together with a commonly used excessive concentration of $1 \mathrm{mM} \mathrm{Ca}^{2+}$ to be able to study $\mathrm{Ca}^{2+}$-dependent conformational differences. The apparent molecular weight of $\sim 70 \mathrm{kDa}$ of the complex indicated a 1:1 stoichiometry, which was confirmed by cross-linking-independent approaches for detection of the intact CaM/segment-A complex, such as native ESI-MS measurements (Figure 2B) and native gel electrophoresis (Figure S5). Notably, the observation of a 1:1 stoichiometry was also in agreement with the results of our previous cosedimentation experiments employing GST-bMunc13-2(367-780) [28]. Taken together, our assays demonstrate that CaM and segment-A of bMunc13-2 form a stoichiometric complex at apparent $\mathrm{Ca}^{2+}$ concentrations in the high nanomolar range, which reflects the $\mathrm{Ca}^{2+}$ levels required for the interaction of full-length ubMunc13-2 and immobilized CaM [52], and overall fits well within the range of presynaptic $\mathrm{Ca}^{2+}$ concentrations observed during synaptic activity [53]. We would like to mention, however, that in our experience the readout particularly from cross-linking-based $\mathrm{Ca}^{2+}$ titration experiments can be considerably assay-dependent and we thus refrain from further elaborating on the $\mathrm{Ca}^{2+}$ sensitivity of the bMunc13-2-CaM interaction by means of exact numbers and potential physiological relevance, but focus on the structural characterization of the bMunc13-2/CaM complex.

\subsection{Structural Characterization of CaM-bMunc13-2 Segment-A Interaction by XL-MS}

After confirming a 1:1 complex between CaM and segment-A, we performed additional cross-linking experiments to obtain more detailed insights into the three-dimensional structure of the CaM/segment-A complex. For this purpose, we applied XL-MS and used a variety of cross-linkers with different reactivities and spacer lengths (Figure 3A) to obtain complementary distance information. In addition to photo-Met, two homobifunctional, amine-reactive cross-linkers, bis(succinimidyl)suberate $\left(\mathrm{BS}^{3}\right)$ and disuccinimidyldibutyl urea (DSBU), as well as the heterobifunctional, amine-/thiol-reactive cross-linker $N$ - $\gamma$-maleimidobutyryloxysulfosuccinimide ester (s-GMBS) were applied. BS ${ }^{3}$ and DSBU contain two $N$-hydroxysuccinimide (NHS) esters that mainly mediate the formation of a covalent bond between primary amines in lysines within a $\mathrm{C}_{\alpha}$-distance of $\sim 30 \AA$ [54] (Figure S3B). S-GMBS connects sulfhydryl groups in cysteines with primary amines in lysines (Figure S3C).

An overview of complex formation between CaM and segment-A with all cross-linkers used in this study is shown in Figure 3B. While the application of all reagents revealed 1:1 complexes at $\sim 70 \mathrm{kDa}$, DSBU and $\mathrm{BS}^{3}$ led to the formation of an additional complex at an apparent molecular weight of $\sim 110 \mathrm{kDa}$. As a segment-A dimer appears at an apparent molecular weight of $130 \mathrm{kDa}$ in SDS-PAGE analysis (Figure S6), the $\sim 110 \mathrm{kDa}$ band most likely represents a CaM/segment-A (2:1) complex. These 2:1 complexes only occurred with homobifunctional amine-reactive reagents of long spacer lengths, which predominantly connect Lys side chains. They were neither observed by cross-linking via photo-Met or the heterobifunctional short-spacer reagent s-GMBS, nor supported by native ESI-MS or gel electrophoresis analyses. These findings underscored the importance of applying various cross-linking reagents with different reactivity and spacer length, and led us to focus our further investigations on 1:1 complexes. 

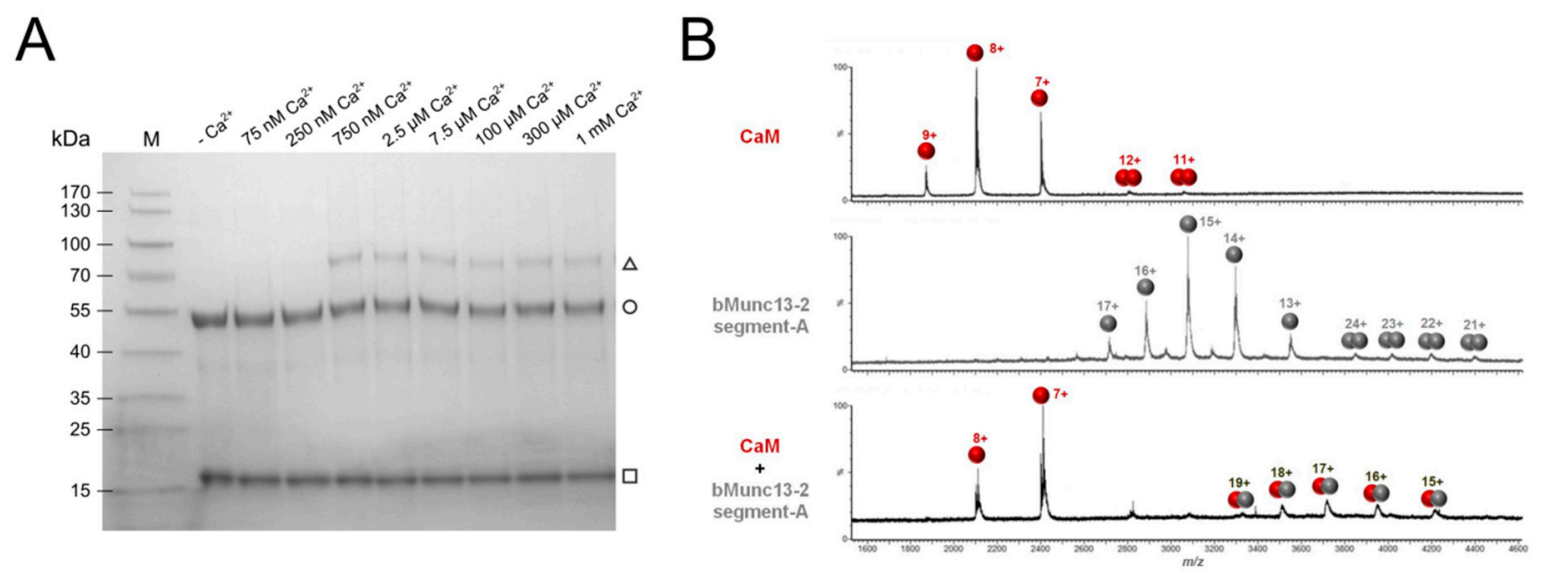

Figure 2. $\mathrm{Ca}^{2+}$-dependent complex formation between CaM and bMunc13-2 segment-A. (A) Gradient (4-20\%) SDS-PAGE gel illustrating cross-linking of the CaM/segment-A complex at various $\mathrm{Ca}^{2+}$ concentrations (adjusted by an EGTA buffer system). Covalent fixation of the complex was achieved by cross-linking of photo-Met residues incorporated in CaM. Proteins are visualized by Coomassie staining. (M) Prestained Protein Ladder, $(\square)$ CaM, $(\bigcirc)$ segment-A and $(\Delta)$ CaM/segment-A complex. (B) Native MS mass spectra of CaM alone (upper panel), segment-A alone (middle panel) and a segment-A/CaM complex (lower panel). Proteins $(10 \mu \mathrm{M})$ were applied in $300 \mathrm{mM}$ ammonium acetate ( $\mathrm{pH} 7$ ) and measurements were performed on a high-mass Q-TOF mass spectrometer. MS data were acquired using a capillary voltage of $1.38 \mathrm{kV}$, a cone voltage of $180 \mathrm{~V}$, and a collision energy of $60 \mathrm{~V}$. Measurements were performed at residual $\mathrm{Ca}^{2+}$ concentration known to be around $5 \mu \mathrm{M}$ [55].

The intense CaM signal observed after cross-linking with photo-Met-CaM was due to the fact that the applied $\mathrm{CaM}$ is a mixture of $\mathrm{CaM}$ species containing none to nine photo-Met residues (variability due to incorporation of photo-Met during expression of CaM in E. coli [43]). Thus, the CaM species without photo-Met did not form a covalent complex with bMunc13-2 through cross-linking and were visible as single proteins in the SDS-PAGE analysis.

To identify the amino acids connected by the different cross-linkers, all SDS-PAGE signals containing the CaM/segment-A (1:1) complex (Figure 3B, triangle) were subjected to in-gel digestion. In addition, in-solution digestion was performed for the photo-Met labeled CaM/segment-A cross-linking reaction mixture. At $1 \mathrm{mM} \mathrm{Ca}^{2+}, 16$ unique intermolecular cross-linking sites between CaM and segment-A were identified with s-GMBS, DSBU, and photo-Met. Half of the cross-links were also detectable at $750 \mathrm{nM} \mathrm{Ca}^{2+}$, which is closer to the physiological concentrations (Figure 3C, upper panel; Table 1). Notably, 10 of 16 cross-links are residing within or flanking the established CaM binding site (aa 719-742) in bMunc13-2.

The highest number of cross-links was obtained with photo-Met-labeled CaM, namely 12 cross-links observed at $1 \mathrm{mM} \mathrm{Ca}^{2+}$ and eight at $750 \mathrm{nM} \mathrm{Ca}^{2+}$ (Table 1), covering six of nine possible photo-Met positions within $\mathrm{CaM}$ for both $\mathrm{Ca}^{2+}$ concentrations. When cross-linking was performed at varying $\mathrm{Ca}^{2+}$ concentrations $(2.5 \mu \mathrm{M}, 45 \mu \mathrm{M}$, and $300 \mu \mathrm{M})$, identical cross-links were observed between CaM and segment-A (Table S1). The two most prevalent cross-links observed in all samples were photo-Met76 in the central $\alpha$-helix of CaM connected with E707 in bMunc13-2 and photo-Met144/145 in the C-terminal lobe of CaM connected with C719 in bMunc13-2 (Table S2, Figure S7).

Analysis of SDS gel bands representing the 1:1 CaM/segment-A complex and monomeric segment-A resulted in the identification of several intramolecular cross-links within segment $A$ (Figure 3C, lower panel; Tables S3-S6). A comparison of these intramolecular cross-links indicated that segment-A adopts similar conformations in the monomeric and in the CaM-bound state at $750 \mathrm{nM}$ and $1 \mathrm{mM} \mathrm{Ca}^{2+}$. Furthermore, analysis of the monomeric segment-A species at $75 \mathrm{nM} \mathrm{Ca}^{2+}$ identified intramolecular cross-links with DSBU and $\mathrm{BS}^{3}$ similar to those observed at higher $\mathrm{Ca}^{2+}$ concentrations (Table S3, Figure S8). For illustration, two exemplary spectra of DSBU cross-links are shown in Figure S9. 
Taken together, our cross-linking data suggest that no major $\mathrm{Ca}^{2+}$-induced structural changes occur in segment-A as both inter- and intramolecular cross-links were found to be highly similar at all $\mathrm{Ca}^{2+}$ concentrations studied.

A

$\mathrm{BS}^{3}$

DSBU

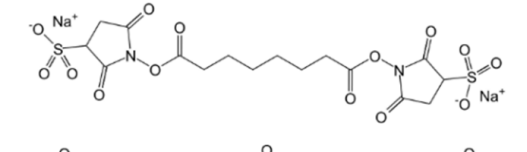

DS

s-GMBS

photo-Met
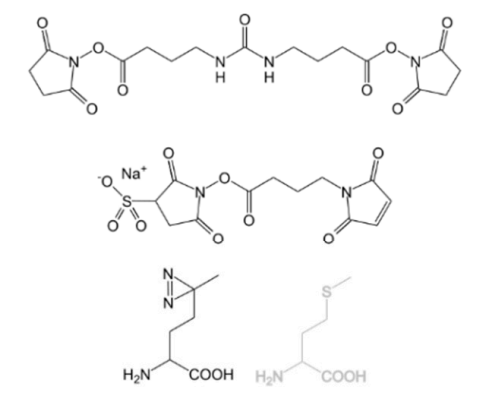

C
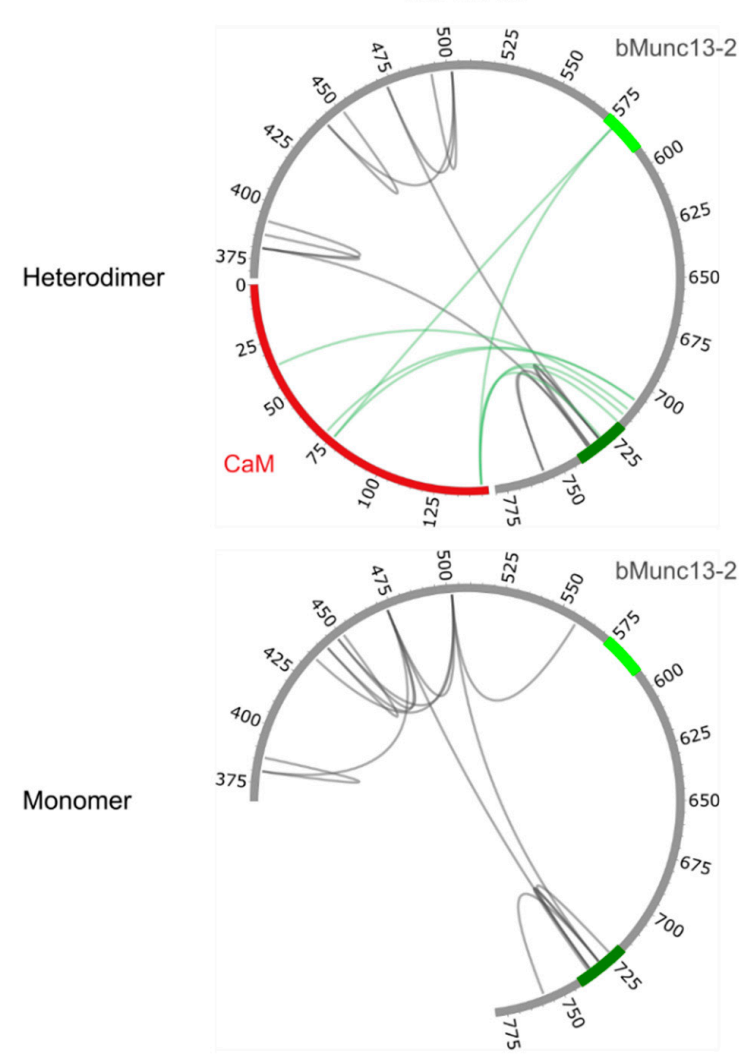
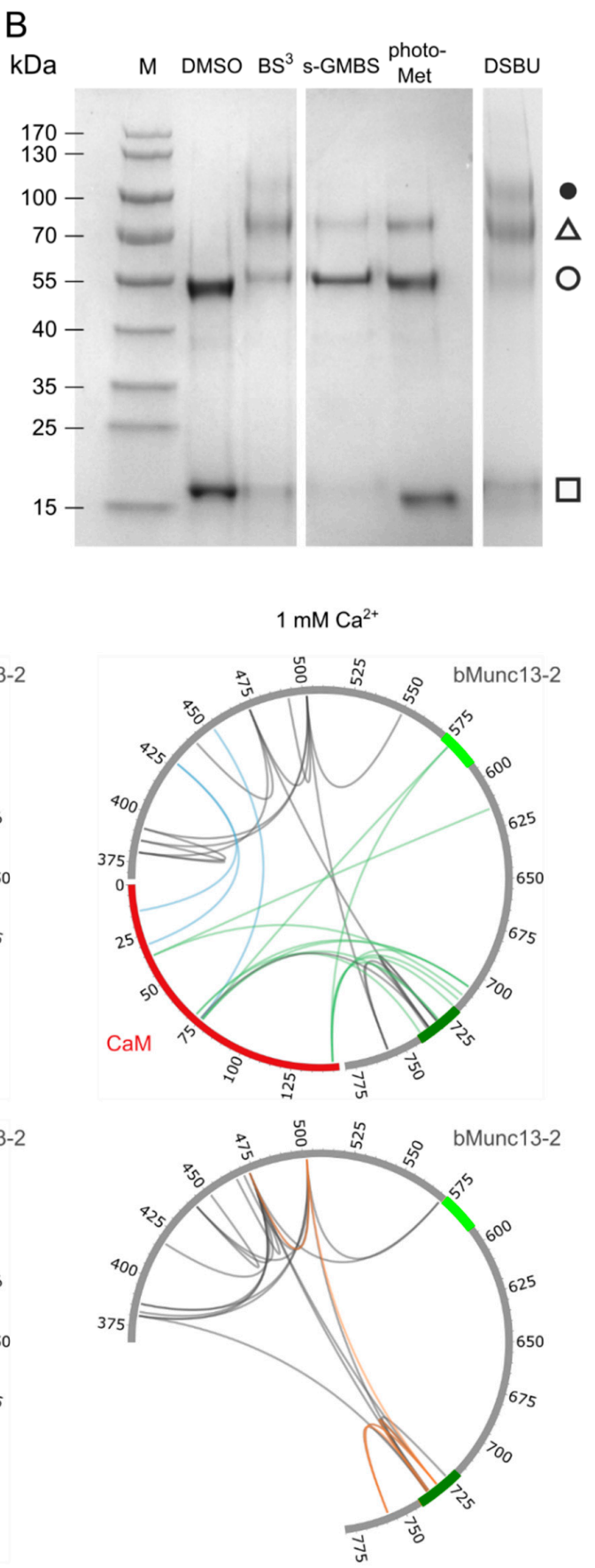

Figure 3. Investigation of $\mathrm{CaM} / \mathrm{bMunc13-2}$ segment-A complex by combining various cross-linking reagents. (A) Structures of applied cross-linking reagents (B) SDS-PAGE (4-20\% gradient gel, Coomassiestained) analysis of complex formation fixed by different cross-linking reagents at $1 \mathrm{mM} \mathrm{Ca}^{2+}$. (M) Prestained Protein Ladder, () CaM, $(\bigcirc)$ segment-A, $(\Delta)$ CaM/segment-A 1:1 complex, $(\bullet)$ potential $\mathrm{CaM} /$ segment-A 2:1 complex. (C) Circos plots showing the identified cross-links with photo-Met (green), s-GMBS (blue), DSBU (dark gray), and BS ${ }^{3}$ (orange). CaM is illustrated in red and segment-A in gray. For cross-links identified with photo-Met at neighboring Met positions, the most probable one is shown. The established (aa 719-742) and predicted (aa 572-594) CaM-binding sites are shown in dark and light green, respectively (see Figure 1). 
Table 1. Intermolecular cross-links identified between CaM and the bMunc13-2 segment- $\mathrm{A}$ at $750 \mathrm{nM}$ and $1 \mathrm{mM} \mathrm{Ca}^{2+}$. $x$ denotes photo-Met; $\mathrm{Y}$, cross-link detected; $\mathrm{N}$, cross-link not detected.

\begin{tabular}{|c|c|c|c|c|}
\hline \multirow{2}{*}{ Cross-Linker } & \multirow{2}{*}{$\begin{array}{c}\text { Cross-Linked } \\
\text { Amino Acid CaM }\end{array}$} & \multirow{2}{*}{$\begin{array}{l}\text { Cross-Linked Amino } \\
\text { Acid Segment-A }\end{array}$} & \multicolumn{2}{|c|}{$\mathrm{Ca}^{2+}$ Concentration } \\
\hline & & & $750 \mathrm{nM}$ & $1 \mathrm{mM}$ \\
\hline \multirow{12}{*}{ Photo-Met } & x36 & D615 & $\mathrm{N}$ & $\mathrm{Y}$ \\
\hline & $x 36$ & E712 & $\mathrm{Y}$ & Y \\
\hline & $x 71 / x 72$ & E707 & Y & Y \\
\hline & $x 71 / x 72$ & E740 & $\mathrm{N}$ & Y \\
\hline & x76 & H578 & $\mathrm{Y}$ & Y \\
\hline & x76 & E707 & Y & Y \\
\hline & $x 76$ & E729 & $\mathrm{N}$ & Y \\
\hline & x144/x145 & H578/H579 & Y & Y \\
\hline & x144/x145 & E707 & $\mathrm{N}$ & $\mathrm{Y}$ \\
\hline & x144/x145 & Y715 & Y & $\mathrm{Y}$ \\
\hline & $\mathrm{x} 144 / \mathrm{x} 145$ & C719 & $\mathrm{Y}$ & $\mathrm{Y}$ \\
\hline & x144/x145 & E729 & Y & Y \\
\hline DSBU & K75 & K735 & $\mathrm{N}$ & $\mathrm{Y}$ \\
\hline \multirow{3}{*}{ s-GMBS } & K75 & C453 & $\mathrm{N}$ & $\mathrm{Y}$ \\
\hline & K13 & C428 & $\mathrm{N}$ & $\mathrm{Y}$ \\
\hline & K30 & C428 & $\mathrm{N}$ & Y \\
\hline
\end{tabular}

\subsection{Inclusion of the C1 Domain of bMunc13-2 Does Not Influence CaM Binding}

To elucidate whether the presence of the C1 domain of bMunc13-2 (aa 853-903) influences bMunc13-2's binding behavior towards CaM, we expressed a C-terminally elongated version of segment-A, which we termed segment-B (aa 367-903; Figure 1 and Figure S2B). We reasoned that the $\mathrm{C} 1$ domain might adopt a defined conformation, stabilizing the structure of segment-B. Cross-linking experiments were conducted with photo-Met-labeled-CaM, confirming the formation of a 1:1 complex in SDS-PAGE analysis (data not shown). In CaM/segment-B complexes, six intermolecular cross-links were identified that were identical to those observed for segment- $A$ in previous experiments (Table 2, Tables S7 and S8) and also point to an interaction of CaM in vicinity to the established binding site of bMunc13-2. This similar cross-link pattern between CaM and the segment-A-specific region (aa 367-780), together with the lack of additional cross-links in the segment-B-specific region (aa 781-903), indicates that the $\mathrm{C} 1$ domain does not influence the structure of the bMunc13-2/CaM complex, at least not to an extent that can be resolved with this method.

Table 2. Comparison of intermolecular cross-links identified between CaM and bMunc13-2 segments. The constraints derived from the cross-links with segment-A and segment-B were used for structural modeling. $x$ denotes photo-Met.

\begin{tabular}{|c|c|c|c|c|}
\hline $\begin{array}{c}\text { Cross-Linked } \\
\text { Amino Acid CaM }\end{array}$ & $\begin{array}{l}\text { Cross-Linked Amino } \\
\text { Acid bMunc13-2 }\end{array}$ & $\begin{array}{l}\text { Segment-A } \\
\text { (aa 367-780) }\end{array}$ & $\begin{array}{l}\text { Segment-B } \\
\text { (aa 367-903) }\end{array}$ & $\begin{array}{l}\text { Segment-C } \\
\text { (aa 703-742) }\end{array}$ \\
\hline x36 & E707 & & & $\checkmark$ \\
\hline x36 & D615 & $\checkmark$ & & \\
\hline x36 & E712 & $\checkmark$ & & $\checkmark$ \\
\hline x71/x72 & E707 & $\checkmark$ & $\checkmark$ & $\checkmark$ \\
\hline$x 71 / x 72$ & E729 & & & $\checkmark$ \\
\hline x71/x72 & E740 & $\checkmark$ & $\checkmark$ & \\
\hline x76 & H578 & $\checkmark$ & & \\
\hline x76 & E707 & $\checkmark$ & $\checkmark$ & $\checkmark$ \\
\hline x76 & E729 & $\checkmark$ & & $\checkmark$ \\
\hline x124 & E729 & & & $\checkmark$ \\
\hline x144/x145 & H578/H579 & 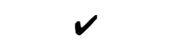 & & \\
\hline x144/x145 & E707 & $\checkmark$ & $\checkmark$ & $\checkmark$ \\
\hline x144/x145 & Y715 & $\checkmark$ & $\checkmark$ & $\checkmark$ \\
\hline x144/x145 & C719 & $\checkmark$ & $\checkmark$ & $\checkmark$ \\
\hline x144/x145 & E729 & $\boldsymbol{v}$ & & $\checkmark$ \\
\hline
\end{tabular}




\subsection{The CaM Binding Site in bMunc13-2 Is N-Terminally Elongated}

Our cross-linking data with segment-A and -B not only confirmed the canonical CaM-binding site of bMunc13-2 (aa 719-742, [28,55]), but also clearly pointed to an involvement of a sequence stretch directly $N$-terminal to that site (see Circos plots in Figure 3C). When we tried to gain further structural insights by molecular modeling, we found that segment-A was not appropriate for docking studies due to its large size and absence of structurally defined domains (data not shown). Therefore, we used our cross-linking data obtained with segment-A for generating models of the complex formed between CaM and segment-C (aa 704-742) (see Figure 1 and Table 3, and details below). Modeling with the ROSETTA software [56] revealed two similar conformations for the CaM/segment-C complex, demonstrating a slight flexibility in the $\mathrm{N}$-terminus of segment-C (Figure 4), which interacts either with aa 71-76 in the central $\alpha$-helix (model A) or the $C$-terminus (model B) of CaM. This flexibility is reflected by the cross-links observed between E707 of segment- $C$ and $x 71 / x 72, x 76$, or $x 144 / x 145$ of $\mathrm{CaM}$ (Figure 4C). While still inconclusive, these models add to what is known about the structure of $\mathrm{CaM} /$ Munc13 peptide complexes so far [16]. According to our models, CaM remains in the compact conformation known for the minimal CaM-binding peptides of all Munc13 isoforms [28] and does not adopt the extended conformation seen with $C$-terminally elongated CaM-binding sites in the highly homologous isoforms Munc13-1 and ubMunc13-2 [32].

A

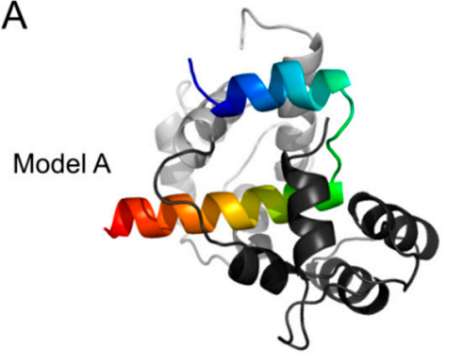

LSLEQVCAETIYINIRCINNEKNVLRERRTROKKLLQELV

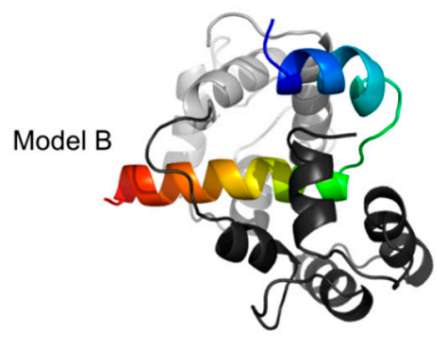

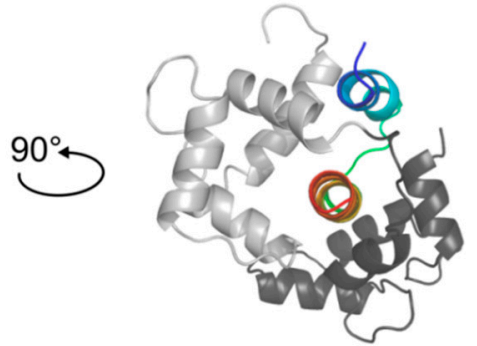

B

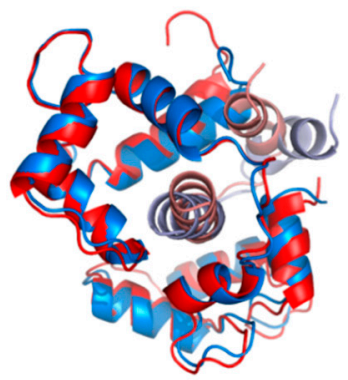

C

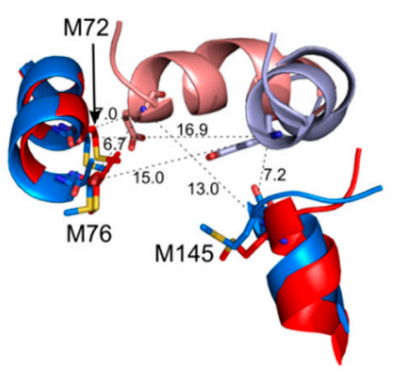

Figure 4. Models of bMunc13-2 segment-C (aa 704-742) with CaM generated by ROSETTA. Segment-C was docked into CaM (PDB-Code: 2O60). The $N$-terminus of bMunc13-2 segment-C interacts with the central $\alpha$-helix (Model A) or the C-terminal amino acids of CaM (Model B). (A) The N-terminal EF-hands of CaM are colored in light gray, the C-terminal EF-hands are colored in dark gray; aa 704-742 of bMunc13-2 are highlighted in rainbow colors starting with blue from the $N$-terminus; the amino acid sequence is given. (B) Superimposition of Model A and B (RMSD of 3.04 $\AA$ ). Red: Model A, blue: Model B; bright colors: segment-C, dark colors: CaM. (C) Zoom into the slight flexibility of the $\mathrm{N}$-terminus in segment-C; aa 704-718 are displayed for segment-C, aa 67-77 and aa 139-148 for CaM. $\mathrm{C}_{\alpha}-\mathrm{C}_{\alpha}$ distances are shown for the cross-links connecting $\mathrm{E} 707$ of segment- $\mathrm{C}$ with the respective Met residues of CaM, which are replaced by photo-Met for cross-linking. Red: Model A, blue: Model B; bright colors: segment-C, dark colors: CaM. 
Table 3. Amino acid sequences of segment- $C$ variants. Summary of segment- $C$ variants employed in the experiments shown in Figures 5 and 6. The replacement by aspartate is colour-coded in the respective variants: green for V709D, blue for I714D, orange for F723D. The canonical CaM binding region [28] is shown in italics; $f$ denotes Bpa.

\begin{tabular}{|c|c|}
\hline Amino Acid Sequences of Segment-C & Variants of Segment-C \\
\hline${ }_{703}$ KLSLEQVCAETIYLNKCINNFKNVLREKRLRQKKLLQELV 742 & Segment-C \\
\hline${ }_{703}$ KLSLEQVCAETIYLNKCINNfKNVLREKRLRQKKLLQELV ${ }_{742}$ & Segment-C-Bpa \\
\hline${ }_{719} C I N N F K N V L R E K R L R Q K K L L Q E L V_{742}$ & Segment-CS \\
\hline${ }_{719}{ }^{C I N N F K N V L R E K R L R Q K K L L Q E L V} V_{742}$ & Segment-CS-Bpa \\
\hline${ }_{719}$ CINNFKNVLREKRLRQKKLLQELVQTASHLSVED ${ }_{742}$ & Segment-CL \\
\hline 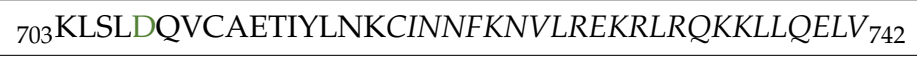 & Segment-C-V709D \\
\hline 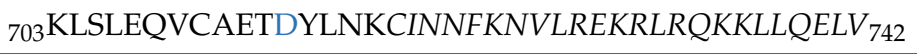 & Segment-C-I714D \\
\hline${ }_{703}{ }_{\text {KLSLEQVCAETIYLNKCINNDKNVLREKRLRQKKLLQELV }}{ }_{742}$ & Segment-C-F723D \\
\hline
\end{tabular}

\subsection{Detailed Analysis of the N-Terminally Elongated Binding Site}

To further corroborate the findings from the modeling approach with experimental data, we performed XL-MS with a synthetic segment-C peptide (aa 703-742, Table 3, Figure S2C). This segment is only 15 amino acids longer than the 24 aa-minimal CaM-binding peptide used previously. Intriguingly, cross-linking experiments with segment- $\mathrm{C}$ revealed that the binding behavior to $\mathrm{CaM}$, as well as the $\mathrm{Ca}^{2+}$ concentrations required for complex formation, were essentially comparable to those found for the substantially larger protein constructs segment-A and segment-B (Figure 5A, compare to Figure 2). The observation that the $\mathrm{Ca}^{2+}$ sensitivity for $\mathrm{CaM}$ binding was identical to that of segment-A was particularly unexpected because of the peptidic nature of segment-C. In accordance with the results from segment-A, the majority of the cross-links between segment-C and photo-Met-CaM (Figure 5B; Table 2, Tables S9 and S10) conclusively pointed to a region $N$-terminal to the known CaM binding motif as the major contact site. Based on these findings, we considered segment- $\mathrm{C}$ as a proxy for the larger protein constructs used so far. When its applicability is justified, as we feel is the case here, such a peptidic proxy increases the methodical range of options for the investigation of protein complexes and we thus relied on segment- $C$ to further characterize the novel CaM-binding site in bMunc13-2.

A

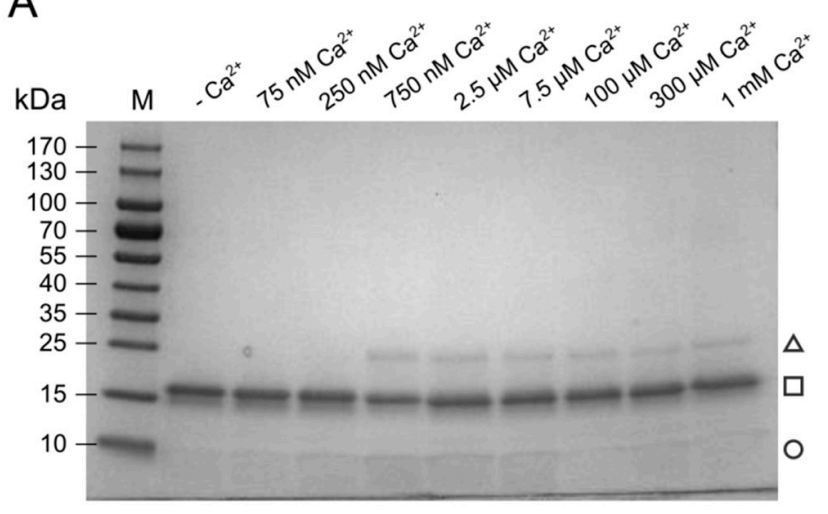

B

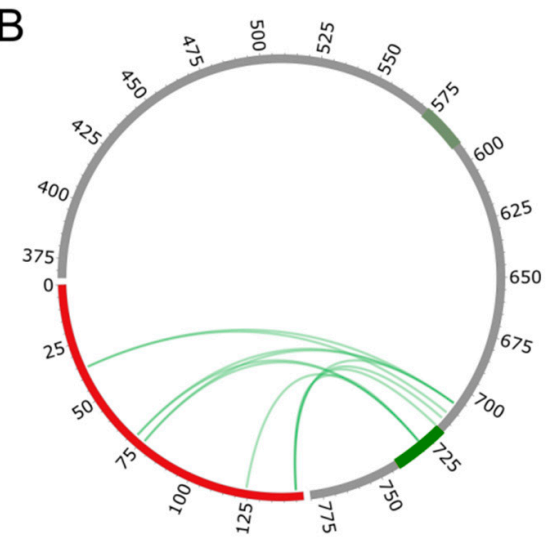

Figure 5. Cont. 
C

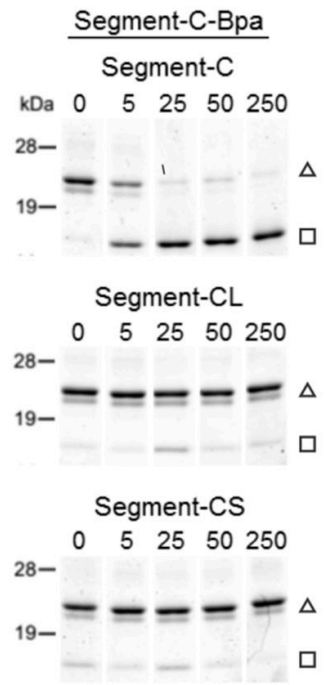

$\mathrm{D}$

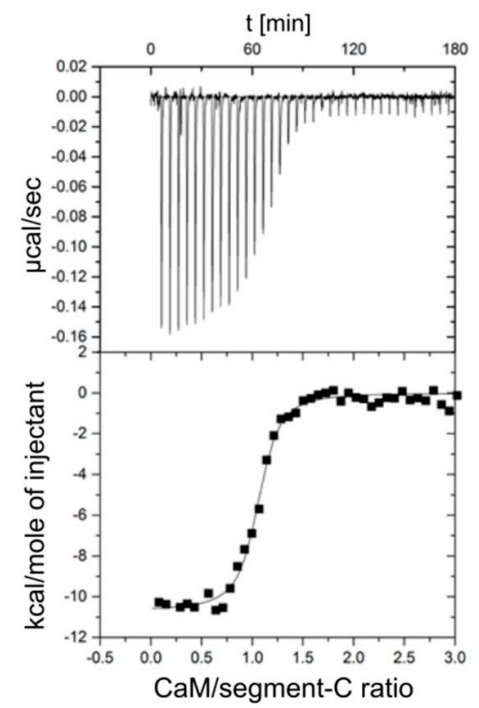

$\mathrm{E}$
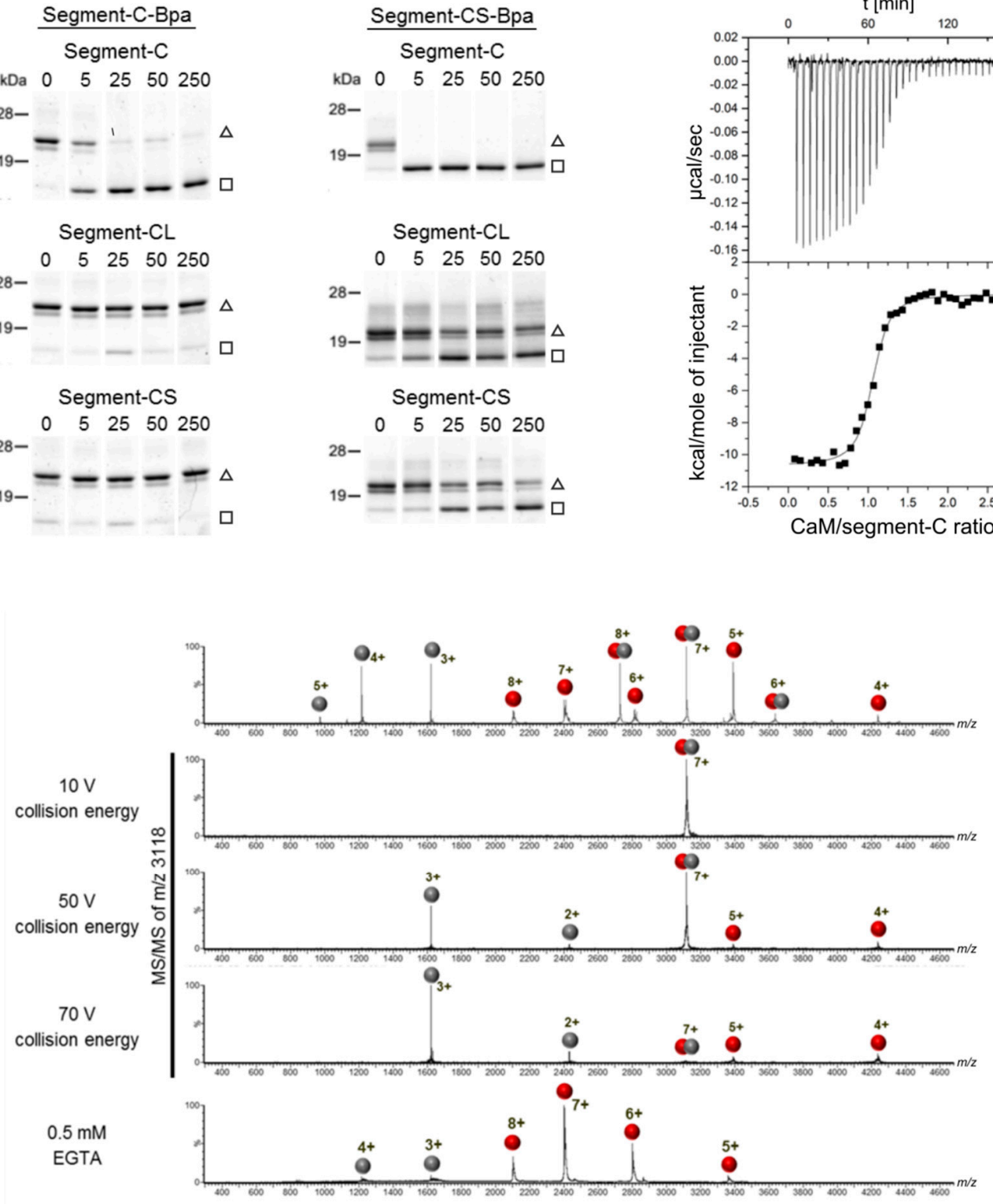

Figure 5. Complex formation between $\mathrm{CaM}$ and the segment-C. (A) $\mathrm{Ca}^{2+}$ concentration required for complex formation. The Coomassie stained gradient (4-20\%) SDS-PAGE gel illustrates cross-linking of the $\mathrm{CaM} /$ segment- $\mathrm{C}$ complex at increasing $\mathrm{Ca}^{2+}$ concentrations. Buffer and cross-linking conditions were identical to Figure 2. The complex was covalently connected by photo-cross-linking via the incorporated photo-Met in CaM. (M) Prestained Protein Ladder, $(\square)$ CaM, $(\bigcirc)$ segment-C and $(\Delta)$ $\mathrm{CaM} /$ segment-C 1:1 complex. (B) Identified cross-links between CaM and segment-C. All cross-links are illustrated as a Circos plot within the whole segment-A for comparison with previous results. CaM is colored in red, segment-A in gray and the binding sites in light and dark green within the segment-A. (C) PAL-based competition assay of segment-C-Bpa and segment-CS-Bpa with segment-C, -CS and -CL. (口) CaM, ( $\Delta$ ) CaM/segment-C-Bpa or CaM/segment-CS-Bpa 1:1 complex. Complete SDS gels and corresponding mass spectra are shown in Figure S10. (D) Affinity measurements. ITC experiments were performed at $6.14 \mu \mathrm{M} \mathrm{Ca}^{2+}$, applying $\mathrm{CaM}$ in the reaction cell and segment- $\mathrm{C}$ in the syringe. A binding event was detected for $\mathrm{N}=1.04, \mathrm{~K}_{\mathrm{D}}=43 \pm 8 \mathrm{nM}, \Delta \mathrm{H}=10.68 \pm 0.17 \mathrm{kcal} \cdot \mathrm{mol}^{-1}$ and $\Delta \mathrm{S}=$ $-2.12 \mathrm{cal} \cdot \mathrm{mol}^{-1} \cdot \mathrm{K}^{-1}$. (E) Native ESI-MS. The ITC sample (CaM and segment-C) was subjected to buffer exchange prior to mass spectrometric analysis. The sample was analyzed in the absence (upper panel) and presence of EGTA (lower panel). MS/MS experiments were performed for the $7+$ charged precursor ion of the complex at $m / z$ 3118. CaM is depicted as red spheres and segment-C as gray spheres. 
We investigated the binding of segment- $\mathrm{C}$ to $\mathrm{CaM}$ with complementary approaches for macromolecular interaction analyses. We first applied a photoaffinity labeling (PAL)-based competition assay $[28,55]$ to compare the CaM-segment-C interaction with our previous results on the minimal CaM-binding bMunc13-2 peptide (aa 719-742, termed Segment-CS hereafter). For this purpose, we generated the photoreactive variants segment-C-Bpa and segment-CS-Bpa (Table 3), in which p-benzoylphenylalanine (Bpa) replaces the phenylalanine at position 723 to enable photo-cross-linking reactions with CaM (Figure S3D) [28,57]. Moreover, we applied segment-CL (aa 719-752) as competitor to mirror the $C$-terminal elongation of the canonical $\mathrm{CaM}$ binding site, which was found in the highly homologous isoforms Munc13-1 and ubMunc13-2 [58]. When segment-C-Bpa was used as photoprobe and unmodified segment- $\mathrm{C}$ as competitor in increasing concentrations, suppression of photoadduct formation was found to start at equimolar concentration and to be virtually complete at a 5-fold molar excess (Figure 5C, upper left panel). In contrast, segment-CS and segment-CL could not suppress photoadduct formation (Figure 5C, middle and lower left panels), indicating that the $\mathrm{N}$-terminal elongation of the CaM-binding site confers a higher affinity to segment-C. Inversely, photoadduct formation of the segment-CS-Bpa photoprobe was completely suppressed already in the presence of equimolar concentrations of segment- $C$ (Figure 5C, upper right panel), indicating that segment- $C$ basically uses the same binding site on $\mathrm{CaM}$ as the minimal binding peptide.

We next applied isothermal titration calorimetry (ITC) to quantify the affinity of segment-C to $\mathrm{CaM}$. These measurements revealed a high affinity with a $\mathrm{K}_{\mathrm{D}}$ value in the lower nanomolar range $(43 \pm 8 \mathrm{nM}$, Figure 5D). Native ESI-MS experiments confirmed this strong interaction as high collision energies of $>50 \mathrm{~V}$ were required to dissociate the complex (Figure 5E). The $\mathrm{Ca}^{2+}$ dependence of the interaction between $\mathrm{CaM}$ and segment- $\mathrm{C}$ was confirmed by dissociating the complex by adding EGTA (Figure 5E, lower panel).

Taken together, our interaction analyses showed that segment- $\mathrm{C}$ binds $\mathrm{CaM}$ with an affinity in the nanomolar range and that this high affinity is conferred to bMunc13-2 by the sequence stretch aa 703-718 located $N$-terminally to the canonical CaM binding site.

\subsection{Hydrophobic Contact Sites in the N-Terminal Part of the bMunc13-2 CaM Binding Sequence Are Crucial} for High-Affinity Binding

To further characterize the involvement of the $\mathrm{N}$-terminal part of segment-C in high-affinity CaM binding, specific mutations were introduced at positions V709, I714 and F723 where potential hydrophobic anchor residues were exchanged to aspartic acid (Table 3). Aspartic acid residues are frequently introduced into CaM target proteins to study their interactions at the molecular level by replacing hydrophobic or basic amino acids in putative CaM binding motifs [59-62].

First, PAL competition assays were performed, in which segment-C-Bpa was used as photoprobe, while segment-C and its mutant variants, V709D, I714D, and F723D, served as competitors (Figure 6A,B). As observed before, a five-fold molar excess of segment- $C$ was sufficient to fully displace its Bpa-labeled counterpart from CaM binding. In contrast, even 50-fold higher concentrations of the mutant variants, V709D and I714D, were not sufficient to reach the same level of displacement. Notably, the variant F723D was not able to displace segment-C-Bpa at all, even not when applied in 50-fold molar excess. Although of a qualitative nature, our data indicate that all three hydrophobic residues exchanged, V709, I714, and F723, contribute to the high CaM-binding affinity of segment-C, with the phenylalanine residue in position 723 obviously functioning as a crucial hydrophobic anchor residue.

To obtain quantitative binding data on the novel CaM binding site and to further characterize the molecular determinants underlying high-affinity binding, surface plasmon resonance (SPR) measurements were performed with segment-C and its mutant variants V709D, I714D, and F723D. For this purpose, biotinylated CaM (Figure S4) was immobilized on a chip and increasing concentrations of segment-C and its variants $(0.1 \mu \mathrm{M}, 0.5 \mu \mathrm{M}$, and $1 \mu \mathrm{M})$ were applied to the CaM-coated surface. The recorded sensograms are shown in Figure $6 \mathrm{C}$. For segment- $C$, a high affinity to $C a M\left(K_{D}=159 n M\right)$ was determined, agreeing well with the ITC data (Figure 5D). Exchanging the hydrophobic residues 
V709 and I714 with aspartic acid resulted in an approximately 3-fold decrease of affinity, while a $K_{D}$ value could not be derived for the F723D variant. Overall, our findings from the competition assays and SPR experiments converged to establish hydrophobic contacts between CaM and the $N$-terminal part of the CaM binding site as crucial determinants of its high binding affinity.

A

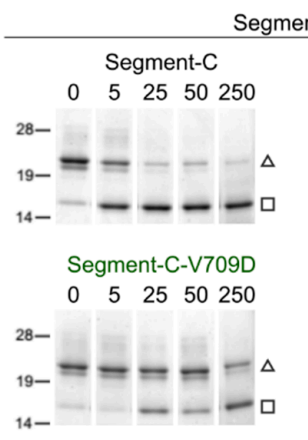

B

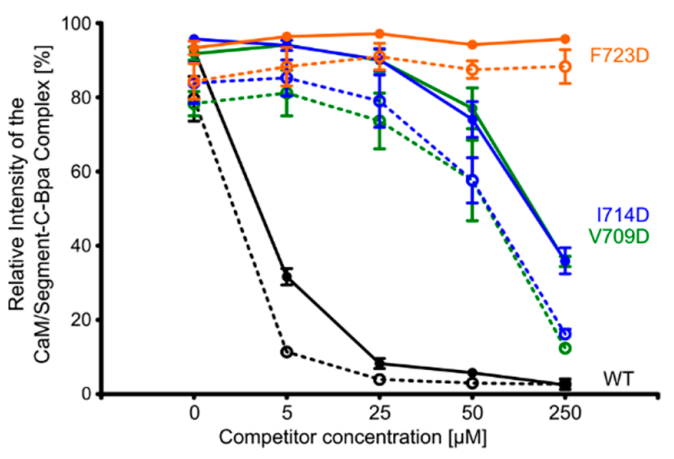

C
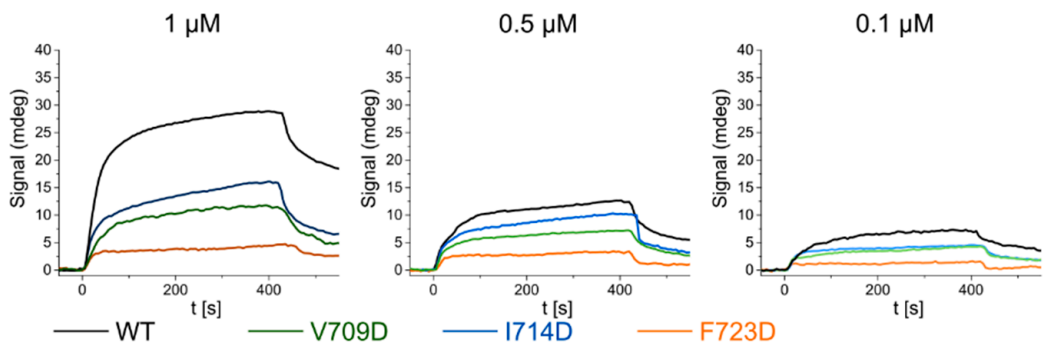

WT
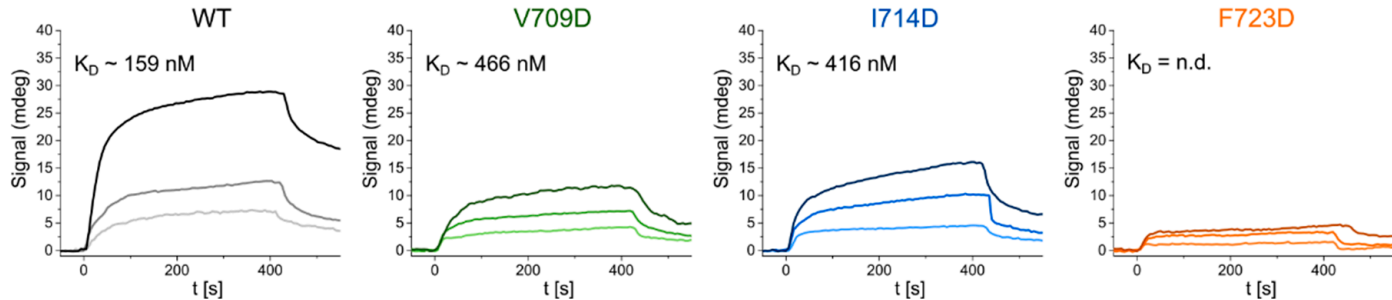

Figure 6. CaM/segment-C interaction studies by photoaffinity labeling and surface plasmon resonance. Amino acid sequences of segment-C variants are given in Table 3. (A,B) PAL-based competition assay of segment-C-Bpa with segment-C and its variants V709D (green), I714D (blue), and F723D (orange). (A) SDS-PAGE analysis of photoreactions; ( $\square)$ CaM, $(\Delta) \mathrm{CaM} /$ segment-C-Bpa 1:1 complex. (B) quantification of photoadduct yield (CaM/segment-C-Bpa signal); solid lines, quantification by SDS-PAGE analysis; dotted lines, quantification by MALDI-TOF-MS. The plot shows the mean of three independent experiments with standard error of mean (SEM) as error bars. A representative set of SDS gels and corresponding mass spectra is shown in Figure S11. (C) SPR measurements of wild-type (WT) and mutated segment-C variants V709D, I714D and F723D. Upper panel: sensograms of all variants for one concentration, lower panel: sensograms of one segment-C variant at different concentrations. $K_{D}$ values are given; n.d.: not determined.

Finally, we tested the significance of amino acid residues V709, I714 and F723 for CaM binding in the protein context. For this purpose, these hydrophobic amino acids were exchanged to aspartic acid in segment-A (Figure 7A). Photo-cross-linking experiments were performed with photo-Met-labeled CaM at $750 \mathrm{nM} \mathrm{Ca}^{2+}$ applying the conditions as described above. The cross-link sites found for segment-A and its mutant variants (Figure 7B, Tables S11-S13) revealed that the cross-linked amino acids are 
located in the region of aa 703-742 in bMunc13-2. However, an increased number of cross-links involving other regions of segment-A were found for the mutant variants in general and for the F723D variant in particular (Figure 7B, Figure S12). These subordinate binding sites included, but were not limited to the further $N$-terminally located candidate CaM binding site in bMunc13-2 (aa 572-594), which was predicted by bioinformatics tools, but shown to be deficient in binding in the protein context [28]. Although we cannot rule out the formation of 2:1 CaM/segment-A complexes when CaM is applied in large excess, we found a predominant occurrence of 1:1 complexes irrespective of the detection method applied (cross-linking-based versus native), which led us to conclude that the predicted binding site is capable of CaM binding only if the high-affinity binding site is compromised. This is the case in the mutant variants due to the absence of hydrophobic anchor residues. Thus, the lower affinity of the variants may lead to conformational promiscuity in the bound state, which is ultimately reflected by scattered cross-linking events.

A

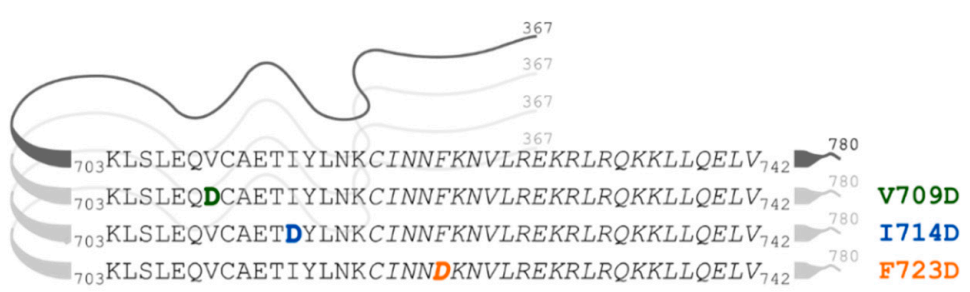

B
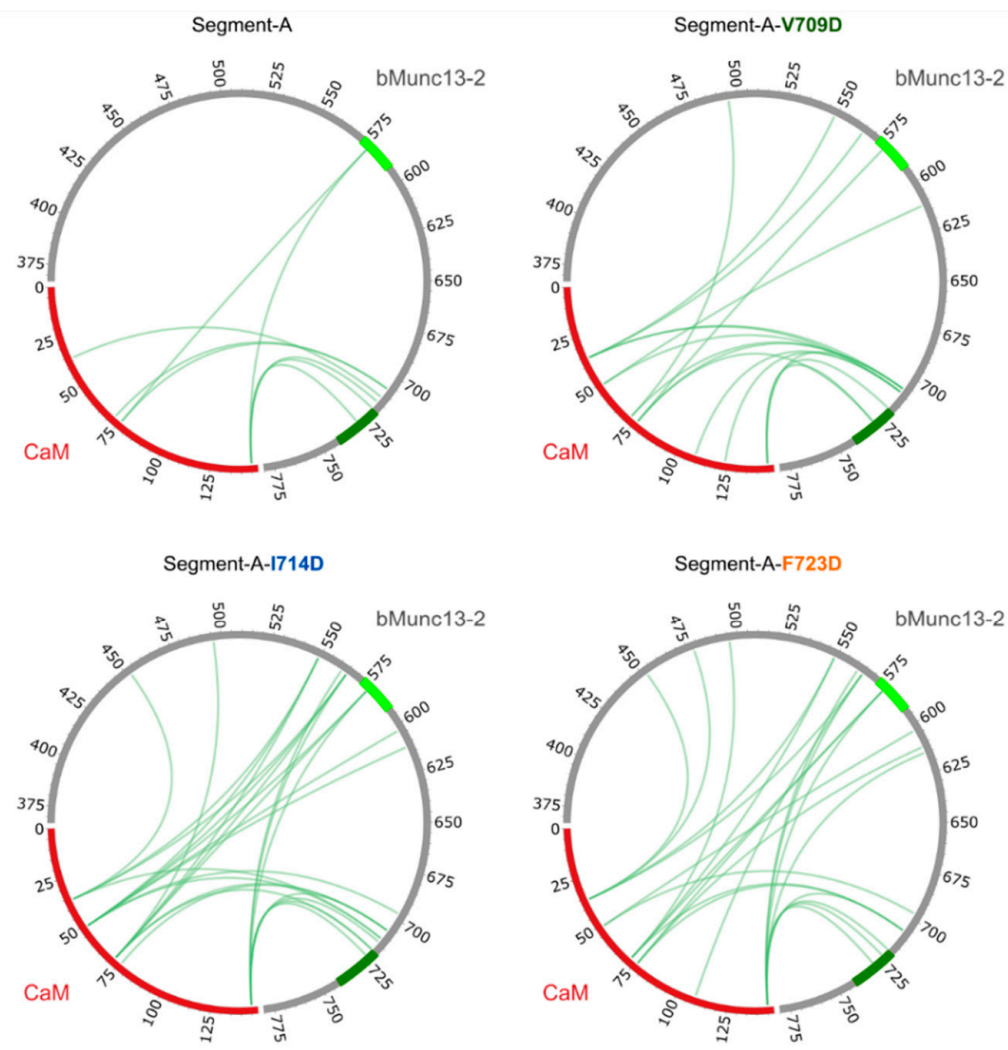

Figure 7. Segment-A variants and photo-Met cross-linking with CaM. (A) Segment-A variants (V709, I714 and F723) were generated by exchanging hydrophobic amino acids to aspartic acid (bold, colored letters). Displayed are the amino acid sequences of the previously employed segment- $C$, in which the proposed CaM binding region is shown in italics. (B) Cross-links observed with photo-Met for wild-type and segment-A variants $\left(750 \mathrm{nM} \mathrm{Ca}^{2+}\right)$. CaM is highlighted in red; CaM binding sites are colored in light green (predicted site, aa 572-594) and dark green (established site, aa 719-742) within segment-A (gray). 


\section{Discussion}

We have previously shown that all four Munc13 isoforms are regulated by $\mathrm{Ca}^{2+} / \mathrm{CaM}$ binding to a CaM-binding site located at a similar position $\mathrm{N}$-terminally of their $\mathrm{C} 1$ domains [28]. While the position of the CaM-binding site appears to be conserved throughout the entire protein family, the type of CaM-binding motif and the structural features of the interaction are not. The most closely related isoforms, Munc13-1 and ubMunc13-2, bind CaM via a C-terminally extended motif of the 1-5-8-26 type [32], but no such detailed information was available for the brain-specific isoform bMunc13-2 exhibiting the strongest CaM-dependent effects on synaptic plasticity in vitro [28].

To study the CaM-bMunc13-2 interaction, we used two different constructs from the $N$-terminal region of bMunc13-2. Both, segment-A (aa 367-780) and segment-B (aa 367-903) formed complexes with $\mathrm{CaM}$ in a 1:1 stoichiometry already at apparent $\mathrm{Ca}^{2+}$ concentrations in the high nanomolar range, rendering these proteins appropriate to structurally characterize $\mathrm{CaM}$ binding behavior under at least pseudo-physiologic conditions: only XL-MS experiments with segment-A revealed the $\mathrm{N}$-terminal extension of the canonical CaM-binding motif in bMunc13-2 (aa 719-742), a unique feature of this Munc13 isoform that had been overlooked in previous experiments with short CaM-binding peptides.

The constraints derived from XL-MS with segment-A served as basis for modeling studies between CaM and segment- $C$ (aa 704-742), a peptide that extends the minimal CaM-binding peptide from bMunc13-2 by 15 aa towards the $N$-terminus and that is predicted to form a straight $\alpha$-helix (Figure $8 \mathrm{~A}$ ). Interestingly, modeling resulted in two similar conformations for the complex due to the flexibility in the positioning of the $\mathrm{N}$-terminus of segment-C. This flexibility is most likely enabled by a kink in the $\alpha$-helix of segment-C, which divides segment- $C$ into an $N$-terminal $(\sim 10-15$ aa) and a $C$-terminal $\alpha$-helical part. Formation of such a kink in the $N$-terminal part of segment- $C$ might be facilitated by a drop in propensity to form a $\alpha$-helix in that region of the peptide, as indicated by lower prediction scores for helix formation (Figure 8A).

The flexible part of segment- $C$ (i.e., the $N$-terminal helix) can either interact with the central $\alpha$-helix or the $C$-terminus of CaM, as reflected by the cross-links observed between E707 of bMunc13-2 and $x 71 / x 72, x 76$, or $x 144 / x 145$ of CaM. Irrespective of this flexibility, the complex between the bMunc13-2 segment- $\mathrm{C}$ and $\mathrm{CaM}$ fails to adopt an extended conformation. This contrasts previous findings for the highly homologous isoforms Munc13-1 and ubMunc13-2 where extended structures were observed for CaM-binding, leading to the definition of the 1-5-8-26 CaM-binding motif [32].

To further characterize the CaM binding site of bMunc13-2, we introduced here the peptide segment-C (aa 703-742) and showed that the $\mathrm{Ca}^{2+}$ sensitivity for CaM binding and the majority of the cross-links were identical to that of the much longer segment-A. Thus, we considered segment- $\mathrm{C}$ as a valid peptidic proxy for the investigation of the CaM binding behavior of bMunc13-2. Indeed, our interaction analyses showed that segment- $\mathrm{C}$ binds $\mathrm{CaM}$ with an affinity in the nanomolar range. Strikingly, this high affinity is conferred to bMunc13-2 by the sequence stretch comprising aa 703-718, i.e., the $N$-terminal extension of the canonical 1-5-10 CaM-binding motif. Specifically, residues V709 and I714, in addition to the putative anchor residue F723, were identified as crucial determinants of high-binding affinity by amino acid exchanges introduced in segment- $\mathrm{C}$ and segment-A. As secondary structure predictions indicated that these amino acid exchanges did not change the overall helicity (data not shown), we conclude that the residues exchanged are indeed hydrophobic contact sites mediating the interaction of segment-C with CaM.

A comparison of the newly identified hydrophobic interaction sites with the canonical 1-5-10 CaM-binding motif (and its closely related variants 1-10, 1-12, and 1-14 identified by a database search), with the 1-5-8-26 motif known from Munc13-1/ubMunc13-2, and with the 1-4-7-8 motif recently discovered in AKAP79 [63], indicates that none of the known or predicted binding motifs can account for the newly identified $N$-terminal contact sites (Figure 8). Additionally, the 1-5-8-26 and 1-4-7-8 CaM-binding motifs differ in their hydrophobic anchor amino acids. In Munc13-1, ubMunc13-2 and AKAP79, the first position of the CaM-binding motif is represented by the bulky, hydrophobic amino acid Trp, while in bMunc13-2, the first position is a Leu or Ile residue. The same applies for 
position 26 within the 1-5-8-26 motif, which in addition is localized differently in comparison to the aligned CaM binding site region of Munc13-1 and ubMunc13-2 (Figure S13, [32]).

A

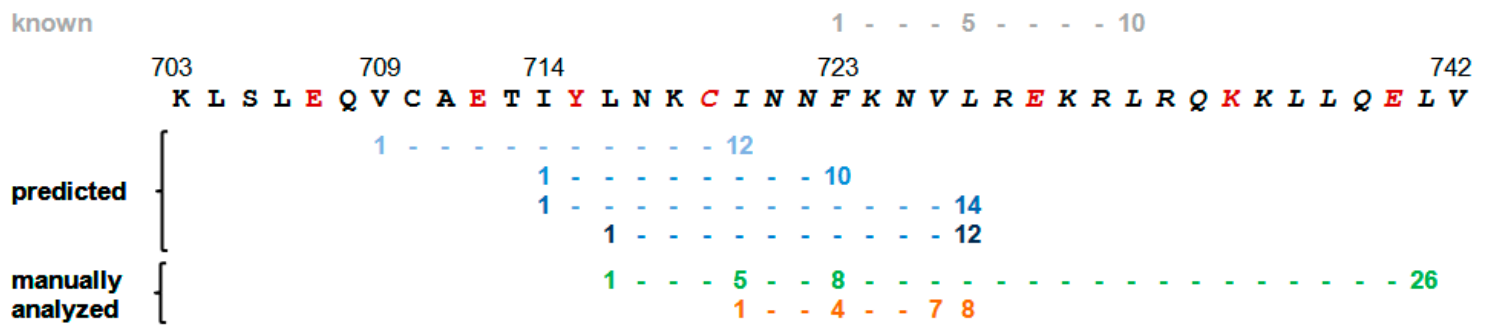

Jnet $\quad--\mathrm{HH} \mathrm{H} \mathrm{H} \mathrm{H} \mathrm{H} \mathrm{H} \mathrm{H} \mathrm{H} \mathrm{H} \mathrm{H} \mathrm{H} \mathrm{H} \mathrm{H} \mathrm{H} \mathrm{H} \mathrm{H} \mathrm{H} \mathrm{H} \mathrm{H} \mathrm{H} \mathrm{H} \mathrm{H} \mathrm{H} \mathrm{H} \mathrm{H} \mathrm{H} \mathrm{H} \mathrm{H}$

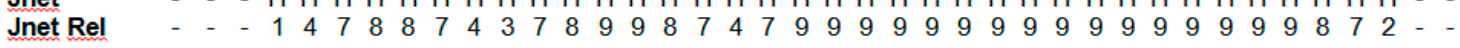

PROF_sec - - $\mathrm{HH} \mathrm{H} \mathrm{H} \mathrm{H} \mathrm{H} \mathrm{H} \mathrm{H} \mathrm{H} \mathrm{H} \mathrm{H} \mathrm{H} \mathrm{H} \mathrm{H} \mathrm{H} \mathrm{H} \mathrm{H} \mathrm{H} \mathrm{H} \mathrm{H} \mathrm{H} \mathrm{H} \mathrm{H} \mathrm{H} \mathrm{H} \mathrm{H} \mathrm{H} \mathrm{H} \mathrm{H}$

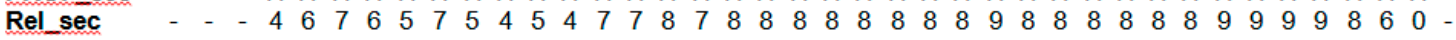
score PSIPRED - - $\mathrm{HH} \mathrm{H} \mathrm{H} \mathrm{H} \mathrm{H} \mathrm{H} \mathrm{H} \mathrm{H} \mathrm{H} \mathrm{H} \mathrm{H} \mathrm{H} \mathrm{H} \mathrm{H} \mathrm{H} \mathrm{H} \mathrm{H} \mathrm{H} \mathrm{H} \mathrm{H} \mathrm{H} \mathrm{H} \mathrm{H} \mathrm{H} \mathrm{H} \mathrm{H} \mathrm{H} \mathrm{H} \mathrm{H}$

B
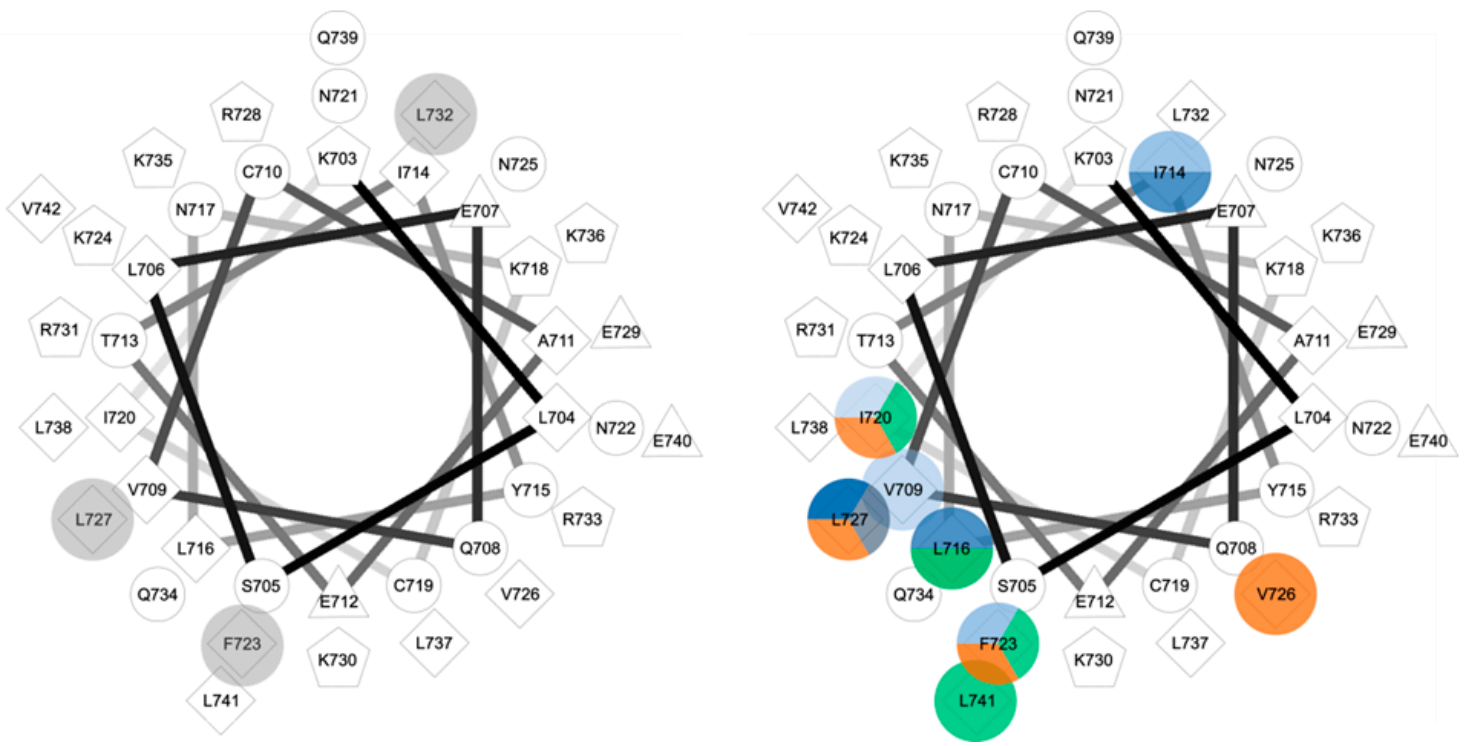

Figure 8. Analysis of potential binding motifs in bMunc13-2 segment-C. Only CaM binding motifs starting $N$-terminally to position 1 of the proposed 1-5-10 binding motif were analyzed. The known 1-5-10 CaM binding motif is colored in gray, CaM binding motifs identified by database comparison are shown in different shades of blue, manually identified CaM binding motifs are colored green and orange. (A) Amino acid sequence, predicted CaM binding motifs and secondary structure prediction of segment-C. Amino acids identified in cross-linking studies are highlighted in red. Three different prediction servers were used: Jpred (www.compbio.dundee.ac.uk/jpred), PredictProtein (www. predictprotein.org), and PSIPRED (bioinf.cs.ucl.ac.uk/psipred). The algorithms Jnet and PROF_sec provide a score for $\alpha$-helical propensity between 0 (lowest propensity) and 9 (highest propensity). $H$, predicted to be $\alpha$-helical. (B) Helical wheel projection of segment-C. The known CaM binding motif is displayed on the left; novel CaM binding motifs are highlighted on the right.

Visualization of the candidate CaM-binding motifs in a helical wheel projection indicates that the potential hydrophobic anchor residues of the 1-5-8-26 and the 1-4-7-8 motifs fall together into neighboring regions at the same side of the $\alpha$-helix, which could indicate an extended conformation of $\mathrm{CaM}$ while binding to bMunc13-2 (Figure 8). In support of our models created for the segment-C/CaM 
complex, an $\alpha$-helix with a kink can be conceived for bMunc13-2, potentially fulfilling the molecular requirement for the extended conformation typical for CaM complexes of the 1-5-8-26 type [32]. However, we did not find any evidence by XL-MS for an extended conformation. Taken together, we consider the 1-5-8-26 motif as unlikely candidate for the CaM-binding motif of bMunc13-2. We then extended the analysis of potential CaM-binding motifs to all Munc13 isoforms and complemented it by secondary structure predictions (Figure S13). Overall, we realized that several candidate CaM binding motifs seem to be compatible with the respective region of bMunc13-2, probably related to its high content of hydrophobic amino acids. Most interesting, however, was the observation of a predicted helical structure starting more $N$-terminally compared to the other Munc13 isoforms. This helix, although predicted to be straight, likely includes a kink as indicated by our models and further supported by a local drop in the predicted propensity for helix formation (Figure 8A). These observations strengthen the existence of a unique $N$-terminally extended $\mathrm{CaM}$ binding motif in bMunc13-2. We thus propose the presence of a novel CaM-binding motif with additional hydrophobic contact sites $N$-terminally of aa 719-742 of bMunc13-2. So far, the positions indicate a 1-6-15 motif, which has to be validated in further studies including high-resolution structural analysis. The fact that the proposed hydrophobic positions (V709, I714, and F723) as well as the secondary structure prediction are conserved from zebrafish to rats to humans and differ from the other isoforms Munc13-1, ubMunc13-2 and Munc13-3 (Figures S14 and S15) further substantiates our finding of a novel CaM binding motif in bMunc13-2.

\section{Conclusions}

We demonstrate that the interaction between $\mathrm{CaM}$ and bMunc13-2 is unique among the Munc13 isoforms and mediated by a novel CaM binding site, which is an $N$-terminal extension of the known CaM 1-5-10 binding motif. Munc13-1 and ubMunc13-2 exhibit a 1-5-8-26 motif [32,64], which is a C-terminal extension of a classical, 1-5-8 CaM binding motif. It remains to be studied whether dimension and type of the CaM-dependent effect on the function of different Munc13 isoforms are related to the mode of CaM interaction via an $\mathrm{N}$ - or a $\mathrm{C}$-terminally extended $\mathrm{CaM}$ binding motif.

Supplementary Materials: The following are available online at http://www.mdpi.com/2073-4409/9/1/136/s1, [65,66]: I, Supplementary Figures S1-S15; II, Supplementary Tables S1-S13; III, Supplementary Methods—Protocol capture page for modeling approach.

Author Contributions: Conceptualization: C.P., N.L., O.J., and A.S.; Investigation: C.P., R.M., C.H.I., A.H., N.L., T.L., J.M., O.J., and A.S.; Methodology: C.P., R.M., C.H.I., A.H., N.L., T.L., J.M., O.J., and A.S.; Visualization: C.P., R.M., C.H.I., A.H., T.L., J.M., O.J., and A.S.; Writing-original draft: C.P., N.L., O.J., and A.S.; Writing-review and editing: C.P., R.M., N.L., J.M., and A.S. All authors have read and agreed to the published version of the manuscript.

Funding: This research was funded by the DFG (project Si 867/15-2) and the region of Saxony-Anhalt. Work in the Meiler laboratory was supported through NIH NIGMS R01 GM080403 and GM073151.

Acknowledgments: The authors would like to thank Michael Götze for continuous improvement of the StavroX and MeroX software tools, Lars van Werven for peptide synthesis, and Xiaohan Wang for assisting with the cross-linking analysis.

Conflicts of Interest: The authors declare no conflict of interest.

\section{References}

1. Carafoli, E.; Krebs, J. Why Calcium? How Calcium Became the Best Communicator. J. Biol. Chem. 2016, 291, 20849-20857. [CrossRef] [PubMed]

2. Berchtold, M.W.; Villalobo, A. The many faces of calmodulin in cell proliferation, programmed cell death, autophagy, and cancer. Biochim. Biophys. Acta 2014, 1843, 398-435. [CrossRef] [PubMed]

3. Chin, D.; Means, A.R. Calmodulin: A prototypical calcium sensor. Trends Cell Biol. 2000, 10, 322-328. [CrossRef]

4. Davis, T.N.; Thorner, J. Vertebrate and yeast calmodulin, despite significant sequence divergence, are functionally interchangeable. Proc. Natl. Acad. Sci. USA 1989, 86, 7909-7913. [CrossRef] [PubMed]

5. Babu, Y.S.; Bugg, C.E.; Cook, W.J. Structure of calmodulin refined at 2.2 A resolution. J. Mol. Biol. 1988, 204, 191-204. [CrossRef] 
6. Fallon, J.L.; Quiocho, F.A. A closed compact structure of native Ca2+-calmodulin. Structure 2003, 11, $1303-1307$. [CrossRef]

7. Zhang, M.; Tanaka, T.; Ikura, M. Calcium-Induced Conformational Transition Revealed by the Solution Structure of Apo Calmodulin. Nat. Struct. Biol. 1995, 2, 758-767. [CrossRef]

8. Shen, X.; Valencia, C.A.; Szostak, J.W.; Dong, B.; Liu, R. Scanning the human proteome for calmodulin-binding proteins. Proc. Natl. Acad. Sci. USA 2005, 102, 5969-5974. [CrossRef]

9. Villarroel, A.; Taglialatela, M.; Bernardo-Seisdedos, G.; Alaimo, A.; Agirre, J.; Alberdi, A.; Gomis-Perez, C.; Soldovieri, M.V.; Ambrosino, P.; Malo, C.; et al. The ever changing moods of calmodulin: How structural plasticity entails transductional adaptability. J. Mol. Biol. 2014, 426, 2717-2735. [CrossRef]

10. Tidow, H.; Nissen, P. Structural diversity of calmodulin binding to its target sites. FEBS J. 2013, 280, 5551-5565. [CrossRef]

11. Rhoads, A.R.; Friedberg, F. Sequence motifs for calmodulin recognition. FASEB J. 1997, 11, 331-340. [CrossRef] [PubMed]

12. Vetter, S.W.; Leclerc, E. Novel aspects of calmodulin target recognition and activation. Eur. J. Biochem. 2003, 270, 404-414. [CrossRef] [PubMed]

13. Bähler, M.; Rhoads, A. Calmodulin signaling via the IQ motif. FEBS Lett. 2002, 513, 107-113. [CrossRef]

14. Mruk, K.; Farley, B.M.; Ritacco, A.W.; Kobertz, W.R. Calmodulation meta-analysis: Predicting calmodulin binding via canonical motif clustering. J. Gen. Physiol. 2014, 144, 105-114. [CrossRef]

15. Yap, K.L.; Kim, J.; Truong, K.; Sherman, M.; Yuan, T.; Ikura, M. Calmodulin target database. J. Struct. Funct. Genom. 2000, 1, 8-14. [CrossRef]

16. Lipstein, N.; Goth, M.; Piotrowski, C.; Pagel, K.; Sinz, A.; Jahn, O. Presynaptic Calmodulin targets: Lessons from structural proteomics. Expert Rev. Proteom. 2017, 14, 223-242. [CrossRef]

17. Ma, C.; Su, L.; Seven, A.B.; Xu, Y.; Rizo, J. Reconstitution of the vital functions of Munc18 and Munc13 in neurotransmitter release. Science 2013, 339, 421-425. [CrossRef]

18. Lai, Y.; Choi, U.B.; Leitz, J.; Rhee, H.J.; Lee, C.; Altas, B.; Zhao, M.; Pfuetzner, R.A.; Wang, A.L.; Brose, N.; et al. Molecular Mechanisms of Synaptic Vesicle Priming by Munc13 and Munc18. Neuron 2017, 95, 591-607. [CrossRef]

19. Imig, C.; Min, S.W.; Krinner, S.; Arancillo, M.; Rosenmund, C.; Sudhof, T.C.; Rhee, J.; Brose, N.; Cooper, B.H. The morphological and molecular nature of synaptic vesicle priming at presynaptic active zones. Neuron 2014, 84, 416-431. [CrossRef]

20. Brose, N.; Hofmann, K.; Hata, Y.; Sudhof, T.C. Mammalian homologues of Caenorhabditis elegans unc-13 gene define novel family of C2-domain proteins. J. Biol. Chem. 1995, 270, 25273-25280. [CrossRef]

21. Koch, H.; Hofmann, K.; Brose, N. Definition of Munc13-homology-domains and characterization of a novel ubiquitously expressed Munc13 isoform. Biochem. J. 2000, 349, 247-253. [CrossRef] [PubMed]

22. Augustin, I.; Betz, A.; Herrmann, C.; Jo, T.; Brose, N. Differential expression of two novel Munc13 proteins in rat brain. Biochem. J. 1999, 337, 363-371. [CrossRef]

23. Breustedt, J.; Gundlfinger, A.; Varoqueaux, F.; Reim, K.; Brose, N.; Schmitz, D. Munc13-2 differentially affects hippocampal synaptic transmission and plasticity. Cereb. Cortex 2010, 20, 1109-1120. [CrossRef] [PubMed]

24. Ishiyama, S.; Schmidt, H.; Cooper, B.H.; Brose, N.; Eilers, J. Munc13-3 superprimes synaptic vesicles at granule cell-to-basket cell synapses in the mouse cerebellum. J. Neurosci. 2014, 34, 14687-14696. [CrossRef]

25. Sakamoto, H.; Ariyoshi, T.; Kimpara, N.; Sugao, K.; Taiko, I.; Takikawa, K.; Asanuma, D.; Namiki, S.; Hirose, K. Synaptic weight set by Munc13-1 supramolecular assemblies. Nat. Neurosci. 2018, 21, 41-49. [CrossRef] [PubMed]

26. Böhme, M.A.; Beis, C.; Reddy-Alla, S.; Reynolds, E.; Mampell, M.M.; Grasskamp, A.T.; Lutzkendorf, J.; Bergeron, D.D.; Driller, J.H.; Babikir, H.; et al. Active zone scaffolds differentially accumulate Unc13 isoforms to tune $\mathrm{Ca}(2+)$ channel-vesicle coupling. Nat. Neurosci. 2016, 19, 1311-1320. [CrossRef]

27. Hu, Z.; Tong, X.J.; Kaplan, J.M. UNC-13L, UNC-13S, and Tomosyn form a protein code for fast and slow neurotransmitter release in Caenorhabditis elegans. eLife 2013, 2, e00967. [CrossRef]

28. Lipstein, N.; Schaks, S.; Dimova, K.; Kalkhof, S.; Ihling, C.; Kolbel, K.; Ashery, U.; Rhee, J.; Brose, N.; Sinz, A.; et al. Nonconserved Ca2+/Calmodulin Binding Sites in Munc13s Differentially Control Synaptic Short-Term Plasticity. Mol. Cell. Biol. 2012, 32, 4628-4641. [CrossRef]

29. Chen, Z.; Cooper, B.; Kalla, S.; Varoqueaux, F.; Young, S.M., Jr. The Munc13 proteins differentially regulate readily releasable pool dynamics and calcium-dependent recovery at a central synapse. J. Neurosci. 2013, 33, 8336-8351. [CrossRef] 
30. Junge, H.J.; Rhee, J.S.; Jahn, O.; Varoqueaux, F.; Spiess, J.; Waxham, M.N.; Rosenmund, C.; Brose, N. Calmodulin and Munc13 form a Ca2+ sensor/effector complex that controls short-term synaptic plasticity. Cell 2004, 118, 389-401. [CrossRef]

31. Lipstein, N.; Sakaba, T.; Cooper, B.H.; Lin, K.H.; Strenzke, N.; Ashery, U.; Rhee, J.S.; Taschenberger, H.; Neher, E.; Brose, N. Dynamic control of synaptic vesicle replenishment and short-term plasticity by $\mathrm{Ca}(2+)$-calmodulin-Munc13-1 signaling. Neuron 2013, 79, 82-96. [CrossRef] [PubMed]

32. Rodriguez-Castaneda, F.; Maestre-Martinez, M.; Coudevylle, N.; Dimova, K.; Junge, H.; Lipstein, N.; Lee, D.; Becker, S.; Brose, N.; Jahn, O.; et al. Modular architecture of Munc13/calmodulin complexes: Dual regulation by Ca2+ and possible function in short-term synaptic plasticity. EMBO J. 2009, 29, 680-691. [CrossRef] [PubMed]

33. Sinz, A. The advancement of chemical cross-linking and mass spectrometry for structural proteomics: From single proteins to protein interaction networks. Expert Rev. Proteom. 2014, 11, 733-743. [CrossRef] [PubMed]

34. Sinz, A. Divide and conquer: Cleavable cross-linkers to study protein conformation and protein-protein interactions. Anal. Bioanal. Chem. 2017, 409, 33-44. [CrossRef]

35. Rappsilber, J. The beginning of a beautiful friendship: Cross-linking/mass spectrometry and modelling of proteins and multi-protein complexes. J. Struct. Biol. 2011, 173, 530-540. [CrossRef]

36. Leitner, A.; Walzthoeni, T.; Kahraman, A.; Herzog, F.; Rinner, O.; Beck, M.; Aebersold, R. Probing native protein structures by chemical cross-linking, mass spectrometry, and bioinformatics. Mol. Cell. Proteom. 2010, 9, 1634-1649. [CrossRef]

37. Petrotchenko, E.V.; Borchers, C.H. Crosslinking combined with mass spectrometry for structural proteomics. Mass Spectrom. Rev. 2010, 29, 862-876. [CrossRef]

38. Young, M.M.; Tang, N.; Hempel, J.C.; Oshiro, C.M.; Taylor, E.W.; Kuntz, I.D.; Gibson, B.W.; Dollinger, G. High throughput protein fold identification by using experimental constraints derived from intramolecular cross-links and mass spectrometry. Proc. Natl. Acad. Sci. USA 2000, 97, 5802-5806. [CrossRef]

39. Lössl, P.; Kölbel, K.; Tänzler, D.; Nannemann, D.; Ihling, C.H.; Keller, M.V.; Schneider, M.; Zaucke, F.; Meiler, J.; Sinz, A. Analysis of Nidogen-1/Laminin gamma1 Interaction by Cross-Linking, Mass Spectrometry, and Computational Modeling Reveals Multiple Binding Modes. PLoS ONE 2014, 9, e112886. [CrossRef]

40. Liu, Z.; Szarecka, A.; Yonkunas, M.; Speranskiy, K.; Kurnikova, M.; Cascio, M. Crosslinking constraints and computational models as complementary tools in modeling the extracellular domain of the glycine receptor. PLoS ONE 2014, 9, e102571. [CrossRef]

41. Hofmann, T.; Fischer, A.W.; Meiler, J.; Kalkhof, S. Protein structure prediction guided by crosslinking restraints-A systematic evaluation of the impact of the crosslinking spacer length. Methods 2015, 89, 79-90. [CrossRef] [PubMed]

42. Müller, M.Q.; Schafer, M.; Dreiocker, F.; Ihling, C.H.; Sinz, A. Cleavable Cross-Linker for Protein Structure Analysis: Reliable Identification of Cross-Linking Products by Tandem MS. Anal. Chem. 2010, 82, 6958-6968. [CrossRef] [PubMed]

43. Piotrowski, C.; Ihling, C.H.; Sinz, A. Extending the cross-linking/mass spectrometry strategy: Facile incorporation of photo-activatable amino acids into the model protein calmodulin in Escherichia coli cells. Methods 2015, 89, 121-127. [CrossRef] [PubMed]

44. Dimova, K.; Kalkhof, S.; Pottratz, I.; Ihling, C.; Rodriguez-Castaneda, F.; Liepold, T.; Griesinger, C.; Brose, N.; Sinz, A.; Jahn, O. Structural insights into the calmodulin-Munc13 interaction obtained by cross-linking and mass spectrometry. Biochemistry 2009, 48, 5908-5921. [CrossRef]

45. Lössl, P.; Sinz, A. Combining Amine-Reactive Cross-Linkers and Photo-Reactive Amino Acids for 3D-Structure Analysis of Proteins and Protein Complexes. Methods Mol. Biol. 2016, 1394, 109-127. [CrossRef]

46. Götze, M.; Pettelkau, J.; Schaks, S.; Bosse, K.; Ihling, C.H.; Krauth, F.; Fritzsche, R.; Kuhn, U.; Sinz, A. StavroX-A software for analyzing crosslinked products in protein interaction studies. J. Am. Soc. Mass Spectrom. 2012, 23, 76-87. [CrossRef]

47. Götze, M.; Pettelkau, J.; Fritzsche, R.; Ihling, C.H.; Schafer, M.; Sinz, A. Automated assignment of MS/MS cleavable cross-links in protein 3D-structure analysis. J. Am. Soc. Mass Spectrom. 2015, 26, 83-97. [CrossRef]

48. Kalkhof, S.; Sinz, A. Chances and pitfalls of chemical cross-linking with amine-reactive N-hydroxysuccinimide esters. Anal. Bioanal. Chem. 2008, 392, 305-312. [CrossRef]

49. Raveh, B.; London, N.; Zimmerman, L.; Schueler-Furman, O. Rosetta FlexPepDock ab-initio: Simultaneous folding, docking and refinement of peptides onto their receptors. PLoS ONE 2011, 6, e18934. [CrossRef] 
50. Valentine, K.G.; Ng, H.L.; Schneeweis, L.; Kranz, J.K.; Frederick, K.K.; Alber, T.; Wand, A.J. Crystal Structure of Calmodulin-Neuronal Nitric Oxide Synthase Complex; RCSB PDB: Piscataway, NJ, USA. [CrossRef]

51. Iacobucci, C.; Gotze, M.; Piotrowski, C.; Arlt, C.; Rehkamp, A.; Ihling, C.; Hage, C.; Sinz, A. Carboxyl-PhotoReactive MS-Cleavable Cross-Linkers: Unveiling a Hidden Aspect of Diazirine-Based Reagents. Anal. Chem. 2018, 90, 2805-2809. [CrossRef]

52. Zikich, D.; Mezer, A.; Varoqueaux, F.; Sheinin, A.; Junge, H.J.; Nachliel, E.; Melamed, R.; Brose, N.; Gutman, M.; Ashery, U. Vesicle priming and recruitment by ubMunc13-2 are differentially regulated by calcium and calmodulin. J. Neurosci. 2008, 28, 1949-1960. [CrossRef] [PubMed]

53. Schneggenburger, R.; Neher, E. Presynaptic calcium and control of vesicle fusion. Curr. Opin. Neurobiol. 2005, 15, 266-274. [CrossRef] [PubMed]

54. Merkley, E.D.; Rysavy, S.; Kahraman, A.; Hafen, R.P.; Daggett, V.; Adkins, J.N. Distance restraints from crosslinking mass spectrometry: Mining a molecular dynamics simulation database to evaluate lysine-lysine distances. Protein Sci. A Publ. Protein Soc. 2014, 23, 747-759. [CrossRef] [PubMed]

55. Dimova, K.; Kawabe, H.; Betz, A.; Brose, N.; Jahn, O. Characterization of the Munc13-calmodulin interaction by photoaffinity labeling. Biochim. Biophys. Acta 2006, 1763, 1256-1265. [CrossRef] [PubMed]

56. Kaufmann, K.W.; Lemmon, G.H.; Deluca, S.L.; Sheehan, J.H.; Meiler, J. Practically useful: What the Rosetta protein modeling suite can do for you. Biochemistry 2010, 49, 2987-2998. [CrossRef] [PubMed]

57. Schwarz, R.; Tanzler, D.; Ihling, C.H.; Sinz, A. Monitoring Solution Structures of Peroxisome ProliferatorActivated Receptor beta/delta upon Ligand Binding. PLoS ONE 2016, 11, e0151412. [CrossRef]

58. Göth, M. Investigation of Protein-Ligand Complexes by Native Mass Spectrometry and Ion Mobility-Mass Spectrometry. Diss. Freie Univ. Berl. 2017. [CrossRef]

59. Osawa, M.; Tokumitsu, H.; Swindells, M.B.; Kurihara, H.; Orita, M.; Shibanuma, T.; Furuya, T.; Ikura, M. A novel target recognition revealed by calmodulin in complex with Ca2+-calmodulin-dependent kinase kinase. Nat. Struct. Biol. 1999, 6, 819-824. [CrossRef]

60. Glaser, P.; Elmaoglou-Lazaridou, A.; Krin, E.; Ladant, D.; Barzu, O.; Danchin, A. Identification of residues essential for catalysis and binding of calmodulin in Bordetella pertussis adenylate cyclase by site-directed mutagenesis. EMBO J. 1989, 8, 967-972. [CrossRef]

61. Tokumitsu, H.; Wayman, G.A.; Muramatsu, M.; Soderling, T.R. Calcium/calmodulin-dependent protein kinase kinase: Identification of regulatory domains. Biochemistry 1997, 36, 12823-12827. [CrossRef]

62. De Diego, I.; Kuper, J.; Bakalova, N.; Kursula, P.; Wilmanns, M. Molecular basis of the death-associated protein kinase-calcium/calmodulin regulator complex. Sci. Signal. 2010, 3, ra6. [CrossRef] [PubMed]

63. Patel, N.; Stengel, F.; Aebersold, R.; Gold, M.G. Molecular basis of AKAP79 regulation by calmodulin. Nat. Commun. 2017, 8, 1681. [CrossRef] [PubMed]

64. Herbst, S.; Maucher, D.; Schneider, M.; Ihling, C.H.; Jahn, O.; Sinz, A. Munc13-like skMLCK variants cannot mimic the unique calmodulin binding mode of Munc13 as evidenced by chemical cross-linking and mass spectrometry. PLoS ONE 2013, 8, e75119. [CrossRef] [PubMed]

65. O'Neil, K.T.; Erickson-Vlitanen, S.; DeGrado, W.F. Photolabeling of calmodulin with basic, amphiphilic alpha-helical peptides containing benzoylphenylalanine. J. Biol. Chem. 1989, 264, 14571-14578.

66. Varoqueaux, F.; Sigler, A.; Rhee, J.S.; Brose, N.; Enk, C.; Reim, K.; Rosenmund, C. Total arrest of spontaneous and evoked synaptic transmission but normal synaptogenesis in the absence of Munc13-mediated vesicle priming. Proc. Natl. Acad. Sci. USA 2002, 99, 9037-9042. [CrossRef]

(C) 2020 by the authors. Licensee MDPI, Basel, Switzerland. This article is an open access article distributed under the terms and conditions of the Creative Commons Attribution (CC BY) license (http://creativecommons.org/licenses/by/4.0/). 